\title{
Facile and Promising Method for Michael Addition of Indole and Pyrrole to Electron-Deficient trans- $\beta$-Nitroolefins Catalyzed by a Hydrogen Bond Donor Catalyst Feist's Acid and Preliminary Study of Antimicrobial Activity
}

\author{
Abdullah M. A. Al Majid, ${ }^{1}$ Mohammad Shahidul Islam, ${ }^{1}$ Assem Barakat, ${ }^{1,2}$ \\ Mohamed H. M. Al-Agamy, ${ }^{3}$ and Mu. Naushad ${ }^{1}$ \\ ${ }^{1}$ Department of Chemistry, Faculty of Science, King Saud University, P.O. Box 2455, Riyadh 11451, Saudi Arabia \\ ${ }^{2}$ Department of Chemistry, Faculty of Science, Alexandria University, P.O. Box 426, Ibrahimia, Alexandria 21321, Egypt \\ ${ }^{3}$ Division of Microbiology, Pharmaceutics Department, College of Pharmacy, King Saud University, P.O. Box 2457, \\ Riyadh 11451, Saudi Arabia \\ Correspondence should be addressed to Mohammad Shahidul Islam; mislam@ksu.edu.sa
}

Received 8 October 2013; Accepted 4 November 2013; Published 16 January 2014

Academic Editors: R. P. Herrera, N. T. Patil, Z. Shao, and L.-W. Xu

Copyright (C) 2014 Abdullah M. A. Al Majid et al. This is an open access article distributed under the Creative Commons Attribution License, which permits unrestricted use, distribution, and reproduction in any medium, provided the original work is properly cited.

\begin{abstract}
The importance of cooperative hydrogen-bonding effects has been demonstrated using novel 3-methylenecyclopropane-1,2dicarboxylic acid (Feist's acid (FA)) as hydrogen bond donor catalysts for the addition of indole and pyrrole to trans- $\beta$-nitrostyrene derivatives. Because of the hydrogen bond donor (HBD) ability, Feist's acid (FA) has been introduced as a new class of hydrogen bond donor catalysts for the activation of nitroolefin towards nucleophilic substitution reaction. It has effectively catalyzed the Michael addition of indoles and pyrrole to $\beta$-nitroolefins under optimum reaction condition to furnish the corresponding Michael adducts in good to excellent yields (up to 98\%). The method is general, atom-economical, convenient, and eco-friendly and could provide excellent yields and regioselectivities. Some newly synthesized compounds were for examined in vitro antimicrobial activity and their preliminary results are reported.
\end{abstract}

\section{Introduction}

In the field of advanced organic synthesis, Michael addition reaction is one of the most powerful tools for carbon-carbon bond construction reactions [1-6]. Nitroolefins are very attractive among the many Michael acceptors because of their strong electron-withdrawing nitro group which could be easily transformed into a wide variety of different functionalities [7-9] that may lead to synthesizing important biologically active building blocks and products [10]. Until now a numerous number of catalysts have been reported in the literature, mainly for the Michael addition reactions of heteroarenes to carbonyls $[11,12]$. Moreover a remarkable number of catalysts have been used to catalyze the Michael addition [13-21] reaction of indoles and pyrrole to trans- $\beta$-nitroolefins; however, most of them were Lewis acids [22-25]. Recently some small organic molecules with anion recognition abilities have provided a great deal of inspiration for the development of hydrogen-bonding catalysis [26-28]; in this context, hydrogen bond donor (HBD) catalysis has received some achievement in exploiting the anion recognition abilities offered by urea, thiourea, and guanidinium functionalities [29-31], silanediols [32, 33], silica gel [34], 2-aminopyridinium ions [35-38], sulfamic acid (SA) [9], dipicolinic acid [26], which were found to be highly potent in catalyzing the Michael addition reaction through hydrogen bonding catalysis $[26$, 
39-47]. Yet such small organic molecules with hydrogen bond donor (HBD) ability received little attention in the context of noncovalent catalysis [48]. Excited by the potential of such hydrogen bond donor small molecule, we have initiated a program in our laboratory dedicated toward pioneering the development of Feist's acid (FA) $[49,50]$ as a new class of catalysts that operate through hydrogen bonding interactions.

Indole and its analogs constitute the active class of compounds possessing wide spectrum of biological activities. A variety of indole derivatives have emerged that possess a range of bioactivities including potent anticancer, antiviral, anti-inflammatory, anti-hypertensive, anti-asthmatic and anti-tubercular properties. Some of these compounds are also known to possess anti-inflammatory and analgesic properties [51-53]. Pyrrole heterocyclic derivatives were reported as having important synthetic and biological activities such as COX-1/COX-2 inhibitors and cytotoxic activity against a variety of marine and human tumor models [54-59].

In this communication, we report initial successes with FA catalysis and its application toward the activation of nitroolefin via hydrogen-bonding mechanistic pathway, which effectively catalyze Michael addition of indoles and pyrrole to nitroolefins under optimum reaction condition to afford the corresponding Michael adducts in good to excellent yields. Some newly synthesized compounds were subjected to in vitro antimicrobial activity.

\section{Experimental}

2.1. General Information. Glassware was oven-dried overnight at $120^{\circ} \mathrm{C}$ before use. Reactions were performed under an inert atmosphere using an argon filled glove box and standard Schlenk-line techniques. All the reactions were monitored by TLC analysis using Merck Silica Gel 60 F-254 thin layer plates. Column chromatography was performed on silica gel 100200 mesh.

2.1.1. Materials. Petroleum ether (PE), hexane, and ethyl acetate for column chromatography were distilled prior to use. $\mathrm{CH}_{2} \mathrm{Cl}_{2}$ and EtOH were distilled from $\mathrm{P}_{2} \mathrm{O}_{5}$ and $\mathrm{Mg}$, respectively, and stored on $4 \AA$ molecular sieves. Tetrahydrofuran, benzene, and toluene were distilled from sodium benzophenone ketyl. Acetonitrile and dimethylformamide were dried by distillation over calcium hydride. Nitroolefins $2(\mathbf{a}-\mathbf{i})$ were prepared according to procedures reported in literature $[60]$.

2.1.2. Instrumentation. NMR spectra were recorded with a Jeol spectrometer at $400 \mathrm{MHz}\left({ }^{1} \mathrm{H}-\mathrm{NMR}\right)$ and $100 \mathrm{MHz}$ $\left({ }^{13} \mathrm{C}-\mathrm{NMR}\right.$.). The chemical shifts ( $\delta$ in ppm) were reported down field from tetramethylsilane (TMS, $\delta$ scale) with the deuterated solvent resonance referenced as internal standard. Elemental analyses were performed on a Perkin Elmer 2400 Elemental Analyzer. IR spectra were obtained using FTIR800 Model. Mass spectrometric analysis was conducted by using ESI mode on AGILENT Technologies 6410-triple quad LC/MS instrument.

2.2. General Procedure for the Michael Addition Reaction of Indole with $\beta$-Nitroolefins Catalyzed by Feist's Acid(GP1).
Indole $4 \quad(50 \mathrm{mg}, \quad 0.425 \mathrm{mmol}), \quad \beta$-nitroolefins $2(\mathbf{a}-\mathbf{i})$ $(0.425 \mathrm{mmol})$, and a catalytic amount of Feist's acid (1) $(6 \mathrm{mg}, 0.084 \mathrm{mmol}, 10 \mathrm{~mol} \%)$ in dry ethanol $(3 \mathrm{~mL})$ were charged into a Schlenk tube under an argon atmosphere. The reaction was then stirred at $50^{\circ} \mathrm{C}$ for $48-72$ hours. The reaction mixture was monitored by TLC until starting material was completely consumed. Then the solvent was removed under vacuum. The crude products were isolated by column chromatography to afford pure Michael adducts (7a-i). The analytical data of the known compounds were in accordance with reported literature [58].

2.2.1. 3-(2-Nitro-1-phenylethyl)-1H-indole (Table 2, Entry 1, 7a). Indole $4(50 \mathrm{mg}, 0.43 \mathrm{mmol})$ and $\beta$-nitrostyrene $2 \mathbf{a}$ $(64 \mathrm{mg}, 0.43 \mathrm{mmol})$ in dry ethanol $(3 \mathrm{~mL})$ were reacted in the presence of Feist's acid (1) (6 mg, $0.084 \mathrm{mmol}, 10 \mathrm{~mol} \%$ ) according to GP1. The product was purified by column chromatography on silica (EtOAc/hexane 1:9) yielded 7a as yellow oil [58] (111 mg, $0.42 \mathrm{mmol}, 97 \%)$. IR (KBr): 3417, $15461375,742,700,587 \mathrm{~cm}^{-1} ;{ }^{1} \mathrm{H}-\mathrm{NMR}\left(\mathrm{CDCl}_{3}, 400 \mathrm{MHz}\right) \delta$ 4.89-4.99 (m, 1H, $\left.\mathrm{CHCH}_{2(\mathrm{a})}\right), 5.02-5.12\left(\mathrm{~m}, 1 \mathrm{H}, \mathrm{CHCH}_{2(\mathrm{~b})}\right)$, 5.15-5.23 (m, 1H, $\mathrm{CHCH}_{2}$ ), 7.01-7.11 (m, 2H, ArH), 7.17-7.24 (m, 1H, ArH), 7.25 (s, 1H, NHCH), 7.26-7.36 (m, 5H, ArH), 7.44 (d, $J=8.0 \mathrm{~Hz}, 1 \mathrm{H}$. ArH), 8.09 (s, 1H, NH of Indole); ${ }^{13} \mathrm{C}-$ $\operatorname{NMR}\left(\mathrm{CDCl}_{3}, 100 \mathrm{MHz}\right): \delta 41.6,79.9,111.4,114.5,119.0,120.0$, 121.7, 122.8, 126.7, 127.7, 127.8, 129.0, 136.6, 139.2; [Anal. Calcd. for $\mathrm{C}_{16} \mathrm{H}_{14} \mathrm{~N}_{2} \mathrm{O}_{2}$ : C, 72.16; H, 5.30; N, 10.52; found: C, 72.37; $\mathrm{H}$, 5.23; N, 10.41]; LC/MS (ESI): $\mathrm{M}^{+}$, found 266.08, $\mathrm{C}_{16} \mathrm{H}_{14} \mathrm{~N}_{2} \mathrm{O}_{2}$ requires 266.11 .

2.2.2. 3-[1-(4-Methylphenyl)-2-nitroethyl]-1H-indole (Table 2, Entry 2, $7 \boldsymbol{b})$. Indole 4 (50 $\mathrm{mg}, 0.43 \mathrm{mmol})$ and $\beta$-nitroolefin 2b (70 $\mathrm{mg}, 0.43 \mathrm{mmol})$ in dry ethanol $(3 \mathrm{~mL})$ were reacted in the presence of Feist's acid (1) (6 mg, $0.084 \mathrm{mmol}, 10 \mathrm{~mol} \%$ ) according to GP1. The product was purified by column chromatography on silica (EtOAc/hexane $1: 9$ ) yielded $7 \mathbf{b}$ as yellow oil [58] (117 mg, $0.42 \mathrm{mmol}, 97.2 \%)$. IR (KBr): 3418, $1547,1377,739 \mathrm{~cm}^{-1}$; ${ }^{1} \mathrm{H}-\mathrm{NMR}\left(\mathrm{CDCl}_{3}, 400 \mathrm{MHz}\right) \delta 2.31(\mathrm{~s}$, $\left.1 \mathrm{H}, \mathrm{CH}_{3}\right), 4.86-4.98\left(\mathrm{~m}, 1 \mathrm{H}, \mathrm{CHCH}_{2(\mathrm{a})}\right), 5.01-5.10(\mathrm{~m}, 1 \mathrm{H}$, $\left.\mathrm{CHCH}_{2(\mathrm{~b})}\right), 5.10-5.20\left(\mathrm{~m}, 1 \mathrm{H}, \mathrm{CHCH}_{2}\right), 6.98-7.02(\mathrm{~m}, 2 \mathrm{H}$, ArH), 7.06-7.25 (m, 5H, ArH), 7.34 (d, J = 8.0 Hz, 1H, ArH), $7.46\left(\mathrm{~d}, J=8.0 \mathrm{~Hz}, 1 \mathrm{H}\right.$. ArH), 8.06 (s, $1 \mathrm{H}, \mathrm{NH}$ of Indole); ${ }^{13} \mathrm{C}-$ NMR $\left(\mathrm{CDCl}_{3}, 100 \mathrm{MHz}\right): \delta$ 21.1, 41.3, 80.0, 111.4, 114.7, 119.0, 120.0, 121.6, 122.7, 126.2, 127.7, 129.7, 136.2, 136.6, 137.3; [Anal. Calcd. for $\mathrm{C}_{17} \mathrm{H}_{16} \mathrm{~N}_{2} \mathrm{O}_{2}$ : C, 72.84; $\mathrm{H}, 5.75 ; \mathrm{N}, 9.99$; found: C, 73.03; H, 5.66; N, 10.11]; LC/MS (ESI): $\mathrm{M}^{+}$, found 280.16, $\mathrm{C}_{17} \mathrm{H}_{16} \mathrm{~N}_{2} \mathrm{O}_{2}$ requires 280.12 .

2.2.3. 3-(1-(4-Methoxyphenyl)-2-nitroethyl)-1H-indole (Table 2, Entry 3, 7c). Indole $4(50 \mathrm{mg}, 0.43 \mathrm{mmol})$ and $\beta$ nitroolefin $2 \mathrm{c}(77 \mathrm{mg}, 0.43 \mathrm{mmol})$ in dry ethanol $(3 \mathrm{~mL})$ were reacted in the presence of Feist's acid (1) $(6 \mathrm{mg}, 0.084 \mathrm{mmol}$, $10 \mathrm{~mol} \%$ ) according to GP1. The product was purified by column chromatography on silica (EtOAc/hexane 1:9) yielded 7c as white solid (107 mg, $0.36 \mathrm{mmol}, 84 \%)$ m.p. $149-151^{\circ} \mathrm{C}$ [Lit [58] m.p. $148-150^{\circ} \mathrm{C}$ ]; IR (KBr): 3373, 1545, 1509, 1458, $1420,1374,1241,1179,1027,742,608,548,525 \mathrm{~cm}^{-1} ;{ }^{1} \mathrm{H}-$ $\mathrm{NMR}\left(\mathrm{CDCl}_{3}, 400 \mathrm{MHz}\right) \delta 3.76\left(\mathrm{~s}, 1 \mathrm{H}, \mathrm{OCH}_{3}\right), 4.84-4.93$ (m, 1H, $\left.\mathrm{CHCH}_{2(\mathrm{a})}\right), 5.00-5.08\left(\mathrm{CHCH}_{2} \mathrm{~m}, 1 \mathrm{H}, \mathrm{CHCH}_{2(\mathrm{~b})}\right)$, 
5.08-5.17 (m, 1H,), $6.84(\mathrm{~d}, J=5.2 \mathrm{~Hz}, 2 \mathrm{H} . \operatorname{ArH}), 7.01(\mathrm{~s}$, 1H, NHCH), 7.02-7.10 (m, 1H, ArH), 7.19-7.28 (m, 3H, ArH), $7.36(\mathrm{~d}, J=8.0 \mathrm{~Hz}, 1 \mathrm{H}$. ArH), 7.44 (d, $J=8.0 \mathrm{~Hz}, 2 \mathrm{H}$. ArH), 8.07 (s, 1H, NH of Indole); ${ }^{13} \mathrm{C}-\mathrm{NMR}\left(\mathrm{CDCl}_{3}, 100 \mathrm{MHz}\right): \delta$ 40.9, 55.3, 80.0, 111.4, 114.3, 119.1, 120.0, 121.5, 122.8, 126.1, 128.9, 131.2, 136.6, 139.3, 158.9; [Anal. Calcd. for $\mathrm{C}_{17} \mathrm{H}_{16} \mathrm{~N}_{2} \mathrm{O}_{3}$ : C, 68.91; H, 5.44; N, 9.45; found: C, 79.09; H, 5.53; N, 9.36]; LC/MS (ESI): $\mathrm{M}^{+}$, found 296.18, $\mathrm{C}_{17} \mathrm{H}_{16} \mathrm{~N}_{2} \mathrm{O}_{2}$ requires 296.12.

2.2.4. 3-(1-(4-Chlorophenyl)-2-nitroethyl)-1H-indole (Table 2, Entry $4,7 \boldsymbol{d})$. Indole $4(50 \mathrm{mg}, 0.43 \mathrm{mmol})$ and $\beta$-nitroolefin 2d (79 $\mathrm{mg}, 0.43 \mathrm{mmol})$ in dry ethanol $(3 \mathrm{~mL})$ were reacted in the presence of Feist's acid (1) (6 mg, $0.084 \mathrm{mmol}, 10 \mathrm{~mol} \%$ ) according to GP1. The product was purified by column chromatography on silica (EtOAc/hexane $1: 9$ ) yielded $\mathbf{7 d}$ as oily liquid (124 mg, $0.42 \mathrm{mmol}, 97.4 \%)$. IR (KBr): 3417, 1548, 1376, $1091,736,423 \mathrm{~cm}^{-1} ;{ }^{1} \mathrm{H}-\mathrm{NMR}\left(\mathrm{CDCl}_{3}, 400 \mathrm{MHz}\right) \delta 4.83-4.96$ $\left(\mathrm{m}, 1 \mathrm{H}, \mathrm{CHCH}_{2(\mathrm{a})}\right), 5.02-5.10\left(\mathrm{~m}, 1 \mathrm{H}, \mathrm{CHCH}_{2(\mathrm{~b})}\right), 5.10-5.22$ (m, 1H, $\mathrm{CHCH}_{2}$ ), 6.96-7.03 (m, 1H. ArH), 7.04-7.12 (m, 1H, ArH), 7.15-7.34 (m, 4H, ArH), 7.34-7.46 (m, 3H, ArH), 8.12 (s, $1 \mathrm{H}, \mathrm{NH}$ of Indole); ${ }^{13} \mathrm{C}-\mathrm{NMR}\left(\mathrm{CDCl}_{3}, 100 \mathrm{MHz}\right): \delta 41.0$, 79.4, 111.6, 114.0, 118.1, 118.9, 120.2, 121.6, 123.0, 126.0, 129.2, 133.5, 136.6, 137.8; [Anal. Calcd. for $\mathrm{C}_{16} \mathrm{H}_{13} \mathrm{ClN}_{2} \mathrm{O}_{2}$ : C, 63.90; $\mathrm{H}, 4.36$; N, 9.31; found: C, 64.11; H, 4.05; N, 9.57]; LC/MS (ESI): $\mathrm{M}^{+} \&[\mathrm{M}+2]^{+}$, found $300.01 \& 302.05, \mathrm{C}_{16} \mathrm{H}_{13} \mathrm{ClN}_{2} \mathrm{O}_{2}$ requires 300.07 .

2.2.5. 3-(1-(4-Bromophenyl)-2-nitroethyl)-1H-indole (Table 2, Entry 6, 7e). Indole $4(50 \mathrm{mg}, 0.43 \mathrm{mmol})$ and $\beta$-nitroolefin $2 \mathbf{e}(98 \mathrm{mg}, 0.43 \mathrm{mmol})$ in dry ethanol $(3 \mathrm{~mL})$ were reacted in the presence of Feist's acid (1) (12 mg, $0.168 \mathrm{mmol}, 20 \mathrm{~mol} \%)$ according to GP1. The product was purified by column chromatography on silica (EtOAc/hexane 1:9) yielded 7e as yellow oil (130 mg, $0.38 \mathrm{mmol}, 88 \%)$. IR (KBr): 3405 , 1539, 1380, 1006, 743, 594, 533, $424 \mathrm{~cm}^{-1} ;{ }^{1} \mathrm{H}-\mathrm{NMR}\left(\mathrm{CDCl}_{3}\right.$, $400 \mathrm{MHz}) \delta 4.86-4.98\left(\mathrm{~m}, 1 \mathrm{H}, \mathrm{CHCH}_{2(\mathrm{a})}\right), 4.99-5.10(\mathrm{~m}, 1 \mathrm{H}$, $\left.\mathrm{CHCH}_{2(\mathrm{~b})}\right), 5.10-5.18\left(\mathrm{~m}, 1 \mathrm{H}, \mathrm{CHCH}_{2}\right), 6.95-7.02(\mathrm{~m}, 1 \mathrm{H}$. ArH), 7.02-7.14 (m, 1H, ArH), 7.15-7.27 (m, 4H, ArH), 7.297.48 (m, 3H, ArH), 8.11 (s, 1H, NH of Indole); ${ }^{13} \mathrm{C}-\mathrm{NMR}$ $\left(\mathrm{CDCl}_{3}, 100 \mathrm{MHz}\right): \delta 41.1,79.3,111.8,113.9,118.9,120.2,121.6$, 123.0, 126.0, 129.6, 132.2, 136.6, 138.3, 140.0; [Anal. Calcd. for $\mathrm{C}_{16} \mathrm{H}_{13} \mathrm{BrN}_{2} \mathrm{O}_{2}$ : C, 55.67; H, 3.80; N, 8.12; found: C, 55.27; $\mathrm{H}$, 3.73; N, 7.98]; LC/MS (ESI): $\mathrm{M}^{+} \&[\mathrm{M}+2]^{+}$, found $344.10 \&$ 346.13, $\mathrm{C}_{16} \mathrm{H}_{13} \mathrm{BrN}_{2} \mathrm{O}_{2}$ requires 344.02.

2.2.6. 3-(1-(4-Nitrophenyl)-2-nitroethyl)-1H-indole (Table 2, Entry 8, 7f). Indole 4 (50 $\mathrm{mg}, 0.43 \mathrm{mmol})$ and $\beta$-nitroolefin $2 \mathbf{f}(83 \mathrm{mg}, 0.43 \mathrm{mmol})$ in dry ethanol $(3 \mathrm{~mL})$ were reacted in the presence of Feist's acid (1) (12 mg, $0.168 \mathrm{mmol}, 20 \mathrm{~mol} \%)$ according to GP1. The product was purified by column chromatography on silica (EtOAc/hexane 1:9) yielded $\mathbf{7 f}$ as yellow oil (94 mg, $0.30 \mathrm{mmol}, 70 \%)$. IR (KBr): 3418, 1549, $1520,1341,713,424 \mathrm{~cm}^{-1} ;{ }^{1} \mathrm{H}-\mathrm{NMR}\left(\mathrm{CDCl}_{3}, 400 \mathrm{MHz}\right) \delta$ 5.00-5.19 (m, 2H, $\left.\mathrm{CHCH}_{2}\right), 5.79-5.96\left(\mathrm{~m}, 1 \mathrm{H}, \mathrm{CHCH}_{2}\right)$, 6.96-7.08 (m, 1H. ArH), 7.08-7.61 (m, 7H. ArH), 7.90 (d, $J=8.0 \mathrm{~Hz}, 1 \mathrm{H}$. ArH), 8.16 (s, 1H, NH of Indole); ${ }^{13} \mathrm{C}-\mathrm{NMR}$ $\left(\mathrm{CDCl}_{3}, 100 \mathrm{MHz}\right): \delta 36.5,80.1,111.5,112.9,118.6,120.4,122.1$, 123.1, 125.2, 126.0, 128.7, 130.0, 133.3, 136.4; [Anal. Calcd. for $\mathrm{C}_{16} \mathrm{H}_{13} \mathrm{~N}_{3} \mathrm{O}_{4}$ : C, 61.73; $\mathrm{H}, 4.21 ; \mathrm{N}, 13.50$; found: $\mathrm{C}, 68.05 ; \mathrm{H}$,
4.42; N, 13.69]; LC/MS (ESI): $\mathrm{M}^{+}$, found 344.08, $\mathrm{C}_{16} \mathrm{H}_{13} \mathrm{~N}_{3} \mathrm{O}_{4}$ requires 311.09 .

2.2.7. 3-(1-(2,4-Dichlorophenyl)-2-nitroethyl)-1H-indole (Table 2, Entry 10, 7g). Indole 4 (50 mg, $0.43 \mathrm{mmol})$ and $\beta$ nitroolefin $2 \mathrm{~g}$ (93 $\mathrm{mg}, 0.43 \mathrm{mmol})$ in dry ethanol $(3 \mathrm{~mL})$ were reacted in the presence of Feist's acid (1) $(12 \mathrm{mg}$, $0.168 \mathrm{mmol}, 20 \mathrm{~mol} \%$ ) according to GP1. The product was purified by column chromatography on silica (EtOAc/hexane $1: 9)$ yielded $7 \mathrm{~g}$ as yellow oil (140 $\mathrm{mg}, 0.42 \mathrm{mmol}, 97.5 \%)$. IR (KBr): $3417,1549,1464,1376,1101,820,738 \mathrm{~cm}^{-1} ;{ }^{1} \mathrm{H}-\mathrm{NMR}$ $\left(\mathrm{CDCl}_{3}, 400 \mathrm{MHz}\right) \delta 4.88-5.01\left(\mathrm{~m}, 2 \mathrm{H}, \mathrm{CHCH}_{2}\right), 5.62-5.75$ (m, 1H, $\mathrm{CHCH}_{2}$ ), 7.03-7.16 (m, 4H. ArH), 7.18-7.27 (m, 1H, ArH), 7.32-7.42 (m, 2H, ArH), 7.45 (s, 1H, ArH), 8.16 (s, 1H, $\mathrm{NH}$ of Indole); ${ }^{13} \mathrm{C}-\mathrm{NMR}\left(\mathrm{CDCl}_{3}, 100 \mathrm{MHz}\right): \delta 37.7,80.0$, $111.5,112.9,118.9,120.3,122.0,123.1,126.0,127.7,129.9,132.2$, 134.0, 134.8, 135.3, 136.6; [Anal. Calcd. for $\mathrm{C}_{16} \mathrm{H}_{12} \mathrm{Cl}_{2} \mathrm{~N}_{2} \mathrm{O}_{2}$ : C, 57.33; H, 3.61; N, 8.36; found: C, 57.08; H, 3.43; N, 8.19]; LC/MS (ESI): $\mathrm{M}^{+} \&[\mathrm{M}+2]^{+}$, found $334.12 \& 336.09, \mathrm{C}_{16} \mathrm{H}_{12} \mathrm{Cl}_{2} \mathrm{~N}_{2} \mathrm{O}_{2}$ requires 334.03 .

2.2.8.3-(1-(2,6-Dichlorophenyl)-2-nitroethyl)-1H-indole (Table 2, Entry 12, 7h). Indole 4 (50 $\mathrm{mg}, 0.43 \mathrm{mmol})$ and $\beta$ nitroolefin $2 \mathbf{h}$ (93 $\mathrm{mg}, 0.43 \mathrm{mmol})$ in dry ethanol $(3 \mathrm{~mL})$ were reacted in the presence of Feist's acid (1) (12 mg, $0.168 \mathrm{mmol}, 20 \mathrm{~mol} \%$ ) according to GP1. The product was purified by column chromatography on silica (EtOAc/hexane $1: 9)$ yielded 7 h yellow oily ( $108 \mathrm{mg}, 0.32 \mathrm{mmol}, 75.2 \%)$. IR (KBr): 3417, 1549, 1464, 1376, 1101, 820, $738 \mathrm{~cm}^{-1} ;{ }^{1} \mathrm{H}-\mathrm{NMR}$ $\left(\mathrm{CDCl}_{3}, 400 \mathrm{MHz}\right) \delta 5.28-5.52\left(\mathrm{~m}, 2 \mathrm{H}, \mathrm{CHCH}_{2}\right), 6.12-6.34$ (m, 1H, $\mathrm{CHCH}_{2}$ ), 6.95-7.10 (m, 1H. ArH), 7.10-7.24 (m, 3H. ArH), 7.24-7.65 (m, 4H. ArH), 8.14 (s, 1H, NH of Indole); ${ }^{13} \mathrm{C}-$ NMR $\left(\mathrm{CDCl}_{3}, 100 \mathrm{MHz}\right): \delta 38.0,78.9,111.4,114.0,115.1,117.8$, 119.0, 120.1, 121.6, 122.5, 126.4, 129.4, 134.2, 136.1; [Anal. Calcd. for $\mathrm{C}_{16} \mathrm{H}_{12} \mathrm{Cl}_{2} \mathrm{~N}_{2} \mathrm{O}_{2}$ : C, 57.33; $\mathrm{H}, 3.61 ; \mathrm{N}, 8.36$; found: C, 57.08; $\mathrm{H}, 3.43$; N, 8.19]; LC/MS (ESI): $\mathrm{M}^{+} \&[\mathrm{M}+2]^{+}$; found 334.09 $\& 336.07, \mathrm{C}_{16} \mathrm{H}_{12} \mathrm{Cl}_{2} \mathrm{~N}_{2} \mathrm{O}_{2}$ requires 334.03.

2.2.9. 3-(1-Ferrocenyl)-2-nitroethyl)-1H-indole (Table 1, Entry $12,7 \mathbf{i})$. Indole $4(50 \mathrm{mg}, 0.43 \mathrm{mmol})$ and $\beta$-nitro styrene $2 \mathbf{i}$ $(118 \mathrm{mg}, 0.43 \mathrm{mmol})$ in dry ethanol $(5 \mathrm{~mL})$ were reacted in the presence of Feist's acid (1) (12 mg, $0.168 \mathrm{mmol}, 20 \mathrm{~mol} \%)$ according to GP1. The product was purified by column chromatography on silica (EtOAc/hexane 1:9) yielded $7 \mathbf{i}$ as oily liquid (78 mg, $0.20 \mathrm{mmol}, 46.4 \%$ ). IR (KBr): 3417, 1549, 1464, 1376, 1101, 820, $738 \mathrm{~cm}^{-1} ;{ }^{1} \mathrm{H}-\mathrm{NMR}\left(\mathrm{CDCl}_{3}, 400 \mathrm{MHz}\right)$ $\delta 4.09(\mathrm{~s}, 5 \mathrm{H}$, protons of $\mathrm{Cp}), 4.11-4.25(\mathrm{~m}, 4 \mathrm{H}$, protons of $\mathrm{Cp}), 4.83-5.89\left(\mathrm{~m}, 1 \mathrm{H}, \mathrm{CpCHCH} \mathrm{CH}_{2}\right), 4.93-5.65(\mathrm{~m}, 2 \mathrm{H}$, $\mathrm{CpCHCH}$ ), 7.01 (s, 1H. ArH), $7.11(\mathrm{t}, J=5.2 \mathrm{~Hz}, 1 \mathrm{H} . \mathrm{ArH})$, $7.18(\mathrm{t}, J=5.2 \mathrm{~Hz}, 1 \mathrm{H}$. ArH), $7.34(\mathrm{~d}, J=8.0 \mathrm{~Hz}, 1 \mathrm{H}$. ArH), 7.57 (d, $J=8.0 \mathrm{~Hz}, 1 \mathrm{H}$. ArH), 8.06 (s, $1 \mathrm{H}, \mathrm{NH}$ of Indole); ${ }^{13} \mathrm{C}-$ NMR $\left(\mathrm{CDCL}_{3}, 100 \mathrm{MHz}\right): \delta$ 36.7, 66.8, 67.7, 68.2, 69.0, 80.6, 111.5, 113.9, 119.1, 119.9, 121.8, 122.5, 126, 129.3; [Anal. Calcd. for $\mathrm{C}_{20} \mathrm{H}_{18} \mathrm{FeN}_{2} \mathrm{O}_{2}$ : C, 64.19; H, 4.84; N, 7.48; found: C, 63.97; H, 4.71; N, 7.59]; LC/MS (ESI): $\mathrm{M}^{+}$, found 374.22, $\mathrm{C}_{20} \mathrm{H}_{18} \mathrm{FeN}_{2} \mathrm{O}_{2}$ requires 374.19 .

2.3. General Procedure for the Michael Addition Reaction of Indole with Pyrrole Catalyzed by Feist's Acid (GP2). Pyrrole 
TABLE 1: Condition optimization of Feist's acid catalysis in the addition of indole ${ }^{a}$ (4) to $\beta$-nitro-styrene (2a).

\begin{tabular}{|c|c|c|c|c|c|}
\hline \multirow[b]{2}{*}{ Entry } & \multirow[b]{2}{*}{ Solvent } & \multicolumn{2}{|c|}{$2 a$} & \multicolumn{2}{|c|}{$\begin{array}{c}7 \mathbf{a} \\
\text { Yield up to } 98 \%\end{array}$} \\
\hline & & $t\left({ }^{\circ} \mathrm{C}\right)$ & Time (h) & Cat. mol\% & Yield $^{\mathrm{b}}(\%)$ \\
\hline 1 & $\mathrm{ACN}$ & 60 & 60 & 10 & 49 \\
\hline 2 & $\mathrm{CH}_{2} \mathrm{Cl}_{2}$ & 40 & 16 & 10 & - \\
\hline 3 & THF & 50 & 72 & 10 & - \\
\hline 4 & $\mathrm{MeOH}$ & 60 & 72 & 10 & 62 \\
\hline 5 & $\mathrm{DMF}$ & 60 & 24 & 10 & 96 \\
\hline 6 & ${ }^{i} \mathrm{PrOH}$ & 60 & 42 & 10 & 98 \\
\hline 7 & $\mathrm{AcOH}$ & 60 & 18 & 10 & 91 \\
\hline 8 & Xylene & 60 & 42 & 10 & 88 \\
\hline 9 & Tolene & 60 & 42 & 10 & 89 \\
\hline 10 & Benzene & 60 & 42 & 10 & 86 \\
\hline 11 & $\mathrm{EtOH}$ & $\mathrm{rt}$ & 120 & 0 & - \\
\hline 12 & $\mathrm{EtOH}$ & 60 & 72 & 0 & - \\
\hline 13 & $\mathrm{EtOH}$ & 40 & 60 & 5 & 59 \\
\hline 14 & $\mathrm{EtOH}$ & 50 & 42 & 10 & 95 \\
\hline 15 & EtOH & 50 & 42 & 15 & 98 \\
\hline 16 & EtOH & 50 & 42 & 20 & 98 \\
\hline
\end{tabular}

${ }^{\mathrm{a}}$ The reactions were performed on an $0.425 \mathrm{mmol}$ scale; ${ }^{\mathrm{b}}$ the isolated yield after column purification.

5 (100 mg, $0.86 \mathrm{mmol}), \beta$-nitrostyrene $2(\mathbf{a}-\mathbf{i})(0.86 \mathrm{mmol})$, and a catalytic amount of Feist's acid (1) (12 mg, $0.168 \mathrm{mmol}$, $10 \mathrm{~mol} \%)$ in dry isopropanol $(7 \mathrm{~mL})$ were charged into a Schlenk tube under an argon atmosphere. The reaction was then stirred at $50^{\circ} \mathrm{C}$ for $24-48$ hours. The reaction mixture was monitored by TLC until starting material was completely consumed. Then the solvent was removed under vacuum. The crude products were isolated by column chromatography to afford pure Michael adducts $\mathbf{8}(\mathbf{a}-\mathbf{i})$ as major region-isomer, $\mathbf{9}(\mathbf{a}-\mathbf{d})$ and $\mathbf{9 h}$ as minor region-isomer.

2.3.1. 2-(2-Nitro-1-phenylethyl)-1H-pyrrole (Table 3, Entry 1, 8a) and 2,5-Bis(2-nitro-1-phenylethyl)-1H-pyrrole (Table 3, Entry 1, 9a). Pyrrole 5 (100 mg, $0.86 \mathrm{mmol})$ and $\beta$-nitrostyrene $2 \mathrm{a}(128 \mathrm{mg}, 0.86 \mathrm{mmol})$ in isopropanol $(7 \mathrm{~mL})$ were reacted in the presence of Feist's acid (1) (12 mg, $0.168 \mathrm{mmol}$, $10 \mathrm{~mol} \%$ ) according to GP2. The products were isolated by column chromatography on silica (EtOAc/hexane $0.5: 9.5$ ) yielded as yellow solid 8a (major region-isomer) (165 mg, $0.74 \mathrm{mmol}, 86.0 \%$ ) and $\mathbf{9 a}$ (minor region-isomer) as yellow oil (40 mg, $0.11 \mathrm{mmol}, 12.7 \%)$. (Major region-isomer 8a): IR (KBr): 3423, 1548, 1376, 723, 703, $524\left(\mathrm{~cm}^{-1}\right) ;{ }^{1} \mathrm{H}-\mathrm{NMR}$ $\left(\mathrm{CDCl}_{3}, 400 \mathrm{MHz}\right) \delta$ 4.69-4.85 (m, 1H, $\left.\mathrm{CHCH}_{2(\mathrm{a})}\right)$, 4.85$4.93\left(\mathrm{~m}, 1 \mathrm{H}, \mathrm{CHCH}_{2(\mathrm{~b})}\right), 4.93-5.02\left(\mathrm{~m}, 1 \mathrm{H}, \mathrm{CHCH}_{2}\right), 6.03-$ 6.10 (m, 1H, ArH), 6.14-6.17 (m, 1H, ArH), 6.64-6.72 (m, 1H. ArH), 7.17-7.26 (m, 2H, ArH), 7.26-7.40 (m, 3H, ArH), 7.84 (s, 1H,NH of pyrrole); ${ }^{13} \mathrm{C}-\mathrm{NMR}\left(\mathrm{CDCl}_{3}, 100 \mathrm{MHz}\right): \delta 43.0$ $\left(\mathrm{CHCH}_{2}\right), 79.6\left(\mathrm{CHCH}_{2}\right), 105.9\left(\mathrm{PyC}_{2}\right), 108.8\left(\mathrm{PyC}_{3}\right), 118.3$ $\left(\mathrm{PyC}_{4}\right), 128.0\left(2 \mathrm{C}, \mathrm{PhC}_{2}\right), 128.24\left(\mathrm{PhC}_{4}\right), 129.0\left(\mathrm{PyC}_{1}\right), 129.3$ $\left(2 \mathrm{C}, \mathrm{PhC}_{3}\right), 138.0\left(\mathrm{PhC}_{1}\right)$; [Anal. Calcd. for $\mathrm{C}_{12} \mathrm{H}_{12} \mathrm{~N}_{2} \mathrm{O}_{2}$ : C,
66.65; H, 5.59; N, 12.96; found: C, 66.45; H, 5.51; N, 13.11]; LC/MS (ESI): $\mathrm{M}^{+}$, found 216.11, $\mathrm{C}_{12} \mathrm{H}_{12} \mathrm{~N}_{2} \mathrm{O}_{2}$ requires 216.09; (minor region-isomer 9a): IR (KBr): 3407, 1548, 1376, 703, $524 \mathrm{~cm}^{-1}$; ${ }^{1} \mathrm{H}-\mathrm{NMR}\left(\mathrm{CDCl}_{3}, 400 \mathrm{MHz}\right) \delta 4.68-4.86(\mathrm{~m}, 4 \mathrm{H}$, $\left.\mathrm{CHCH}_{2}\right), 4.86-4.98\left(\mathrm{~m}, 2 \mathrm{H}, \mathrm{CHCH}_{2}\right), 5.98(\mathrm{~d}, \mathrm{~J}=2.2 \mathrm{~Hz}$, 2H, pyrrole), 7.05-7.18 (m, 4H, ArH), 7.18-7.39 (m, 6H, ArH), 7.54 (s, $1 \mathrm{H}, \mathrm{NH}$ of pyrrole); ${ }^{13} \mathrm{C}-\mathrm{NMR}\left(\mathrm{CDCl}_{3}, 100 \mathrm{MHz}\right): \delta$ $42.9\left(\mathrm{CHCH}_{2}\right), 79.2\left(\mathrm{CHCH}_{2}\right), 106.2\left(\mathrm{PyC}_{2}\right), 106.6\left(\mathrm{PyC}_{2}\right)$, $127.85\left(\mathrm{PhC}_{2}\right), 127.92\left(\mathrm{PhC}_{4}\right), 128.24\left(\mathrm{PhC}_{3}\right), 129.30\left(\mathrm{PyC}_{1}\right)$, $138.1\left(\mathrm{PhC}_{1}\right.$ ); [Anal. Calcd. for $\mathrm{C}_{20} \mathrm{H}_{19} \mathrm{~N}_{3} \mathrm{O}_{4}$ : C, 65.74; $\mathrm{H}, 5.24$; $\mathrm{N}, 11.50$; found: C, 66.03; H, 5.37; N, 11.69]; LC/MS (ESI): $\mathrm{M}^{+}$, found $365.13, \mathrm{C}_{12} \mathrm{H}_{12} \mathrm{~N}_{2} \mathrm{O}_{2}$ requires 365.14.

2.3.2. 2-(2-Nitro-1-(p-tolyl)ethyl)-1H-pyrrole (Table 3, Entry 2, $8 \boldsymbol{b})$ and 2,5-Bis(2-nitro-1-(p-tolyl)ethyl)-1H-pyrrole (Table 3, Entry 2, 9b). Pyrrole 5 (100 mg, $0.86 \mathrm{mmol})$ and $\beta$-nitroolefin $2 \mathbf{b}$ (140 mg, $0.86 \mathrm{mmol})$ in isopropanol $(7 \mathrm{~mL})$ were reacted in the presence of Feist's acid (1) $(12 \mathrm{mg}, 0.168 \mathrm{mmol}$, $10 \mathrm{~mol} \%$ ) according to GP2. The products were isolated by column chromatography on silica (EtOAc/hexane $0.5: 9.5$ ) yielded as oily liquid $\mathbf{8 b}$ (major region-isomer) (100 mg, $0.43 \mathrm{mmol}, 51 \%$ ) and $\mathbf{9 b}$ (minor region-isomer) as yellow oil (70 mg, $0.18 \mathrm{mmol}, 21 \%$ ). (Minor region-isomer $\mathbf{8 b}$ ): IR ( $\mathrm{KBr})$ : $3388,1542,1378,716,584 \mathrm{~cm}^{-1} ;{ }^{1} \mathrm{H}-\mathrm{NMR}\left(\mathrm{CDCl}_{3}, 400 \mathrm{MHz}\right)$ $\delta 2.25\left(\mathrm{~s}, 1 \mathrm{H}, \mathrm{CH}_{3}\right), 4.67-4.73\left(\mathrm{~m}, 1 \mathrm{H}, \mathrm{CHCH}_{2(\mathrm{a})}\right), 4.73-4.81$ $\left(\mathrm{m}, 1 \mathrm{H}, \mathrm{CHCH}_{2(\mathrm{~b})}\right), 4.82-4.93\left(\mathrm{~m}, 1 \mathrm{H}, \mathrm{CHCH}_{2}\right), 5.99(\mathrm{~s}, 1 \mathrm{H}$, ArH), 6.07-6.13 (m, 1H, ArH), 6.58-6.59 (m, 1H. ArH), 7.027.05 (m, 2H, ArH), 7.05-7.08 (m, 2H, ArH), 7.73 (s, 1H, NH of pyrrole); ${ }^{13} \mathrm{C}-\mathrm{NMR}\left(\mathrm{CDCl}_{3}, 100 \mathrm{MHz}\right): \delta 21.2\left(\mathrm{CH}_{3}\right), 42.7$ 
TABLE 2: Michael addition of indole (4) to nitroolefins 2(a-i) catalyzed by Feist's acid.

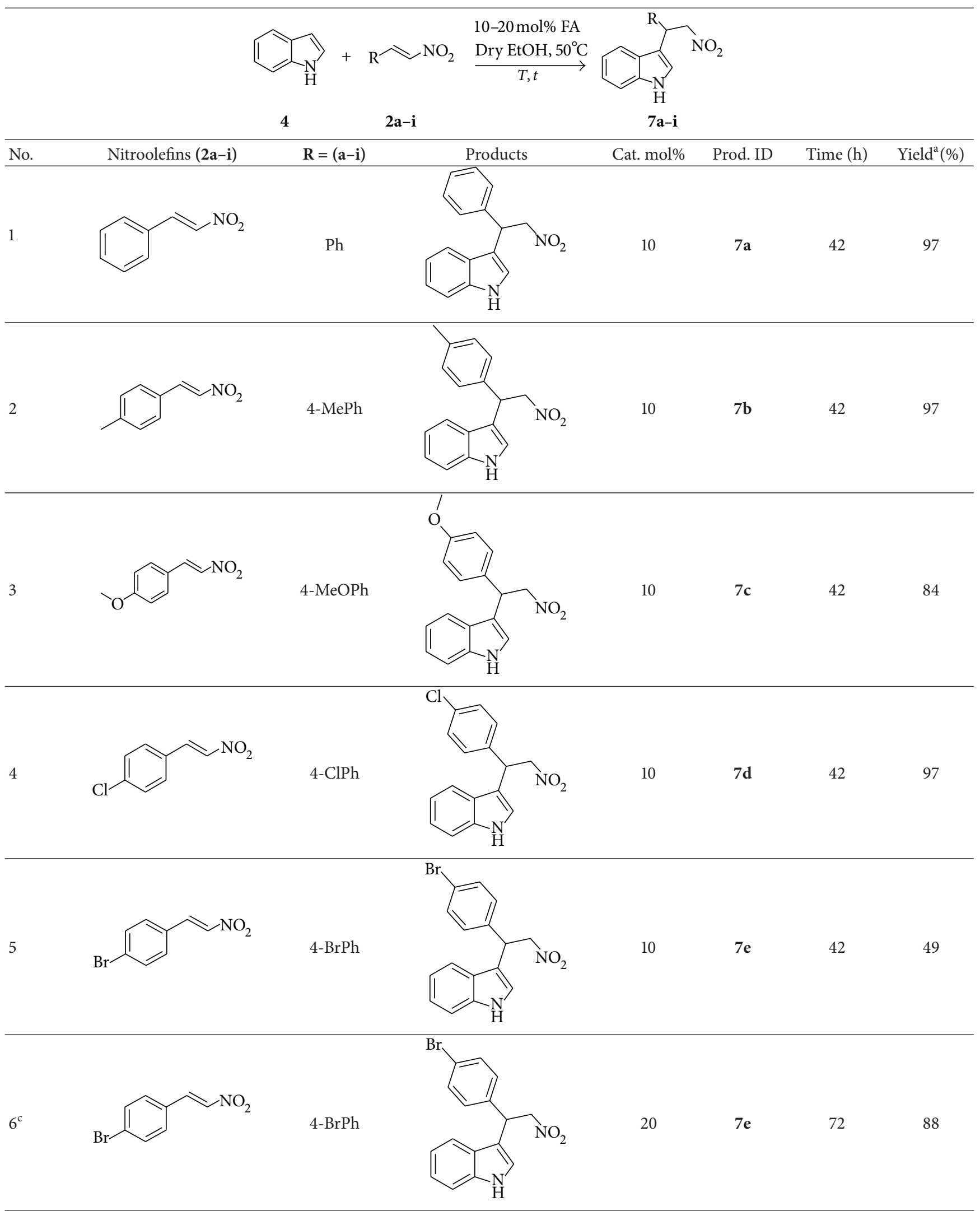


TABLe 2: Continued.

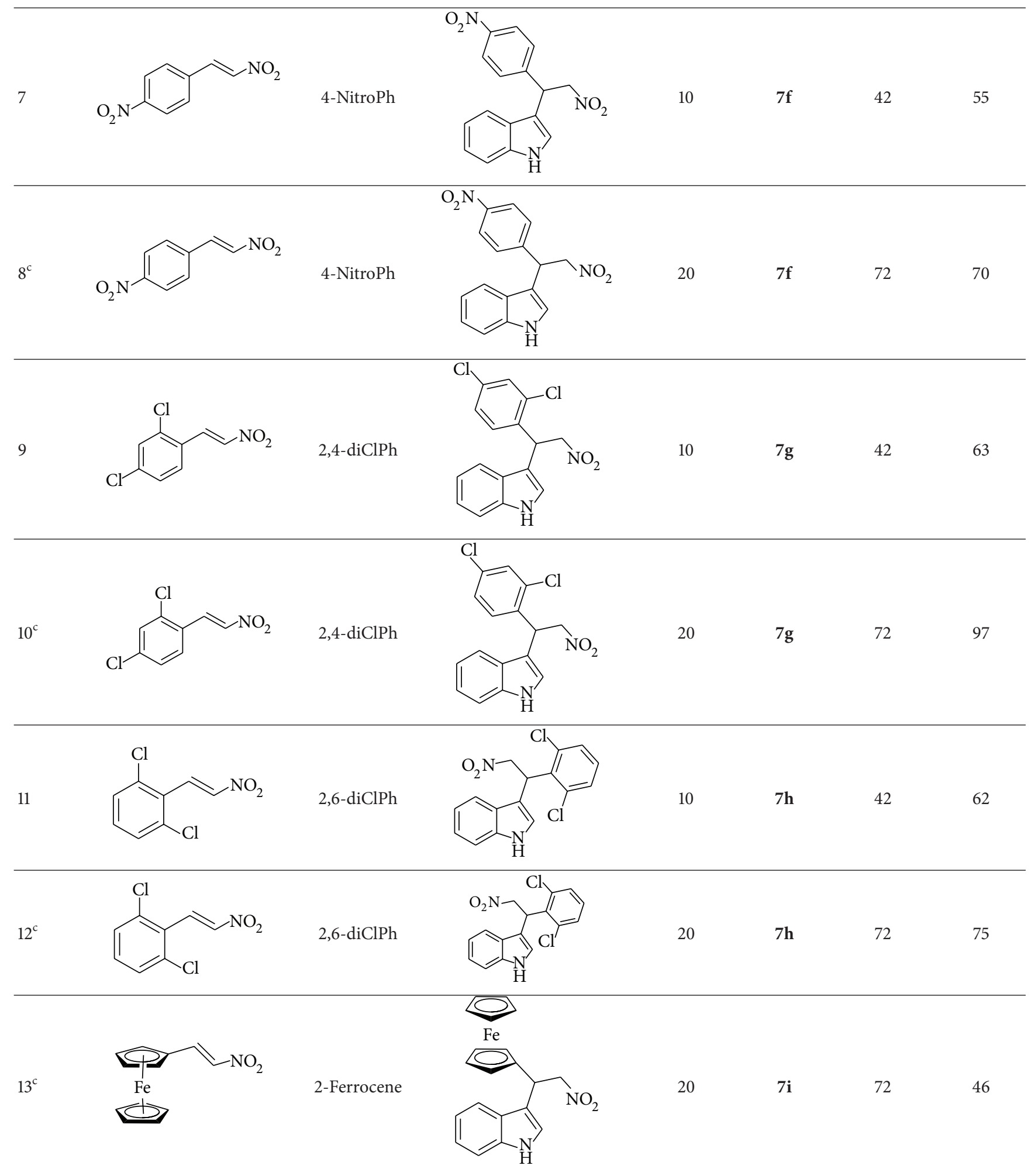

${ }^{a}$ The reactions were performed on $0.425 \mathrm{mmol} \mathrm{scale;}$; the isolated yield after column purification; ${ }^{c} 20 \mathrm{~mol} \%$ catalyst was used and reactions were run for 72 hours. 
TABLE 3: Feist's acid catalysis substrate scope ${ }^{\mathrm{a}}$, pyrrole 5.

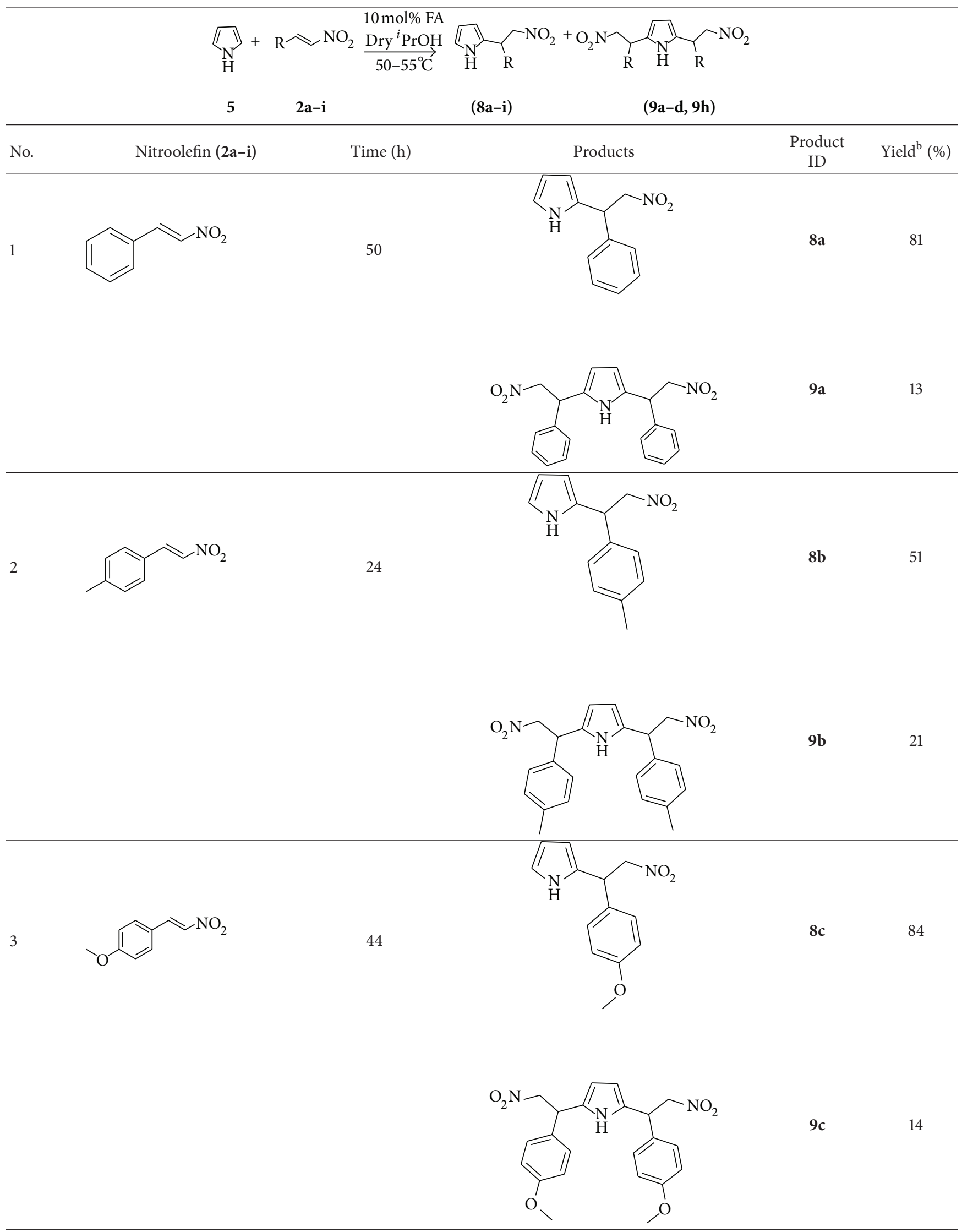


TABle 3: Continued.

4<smiles>O=[N+]([O-])C=Cc1ccc(Cl)cc1</smiles>

20<smiles>O=[N+]([O-])CC(c1ccc(Cl)cc1)c1ccc[nH]1</smiles><smiles>O=[N+]([O-])CC(c1ccc(Cl)cc1)c1ccc(C(C[N+](=O)[O-])c2ccc(Cl)cc2)[nH]1</smiles>

20<smiles>O=[N+]([O-])CC(c1ccc(Br)cc1)c1ccc[nH]1</smiles>

20<smiles>O=NCC(c1ccc([N+](=O)[O-])cc1)c1ccc[nH]1</smiles>

20<smiles>O=[N+]([O-])CC(c1ccc[nH]1)c1ccc(Cl)cc1Cl</smiles>

$8 \mathrm{~g}$

$8 f$

99<smiles>O=[N+]([O-])CC(c1ccc[nH]1)c1ccccc1Cl</smiles>

11

9d

$8 e$ 48
7<smiles>O=[N+]([O-])C=Cc1ccc(Cl)cc1Cl</smiles>

20<smiles>O=[N+]([O-])C=Cc1c(Cl)cccc1Cl</smiles> 
TABLE 3: Continued.

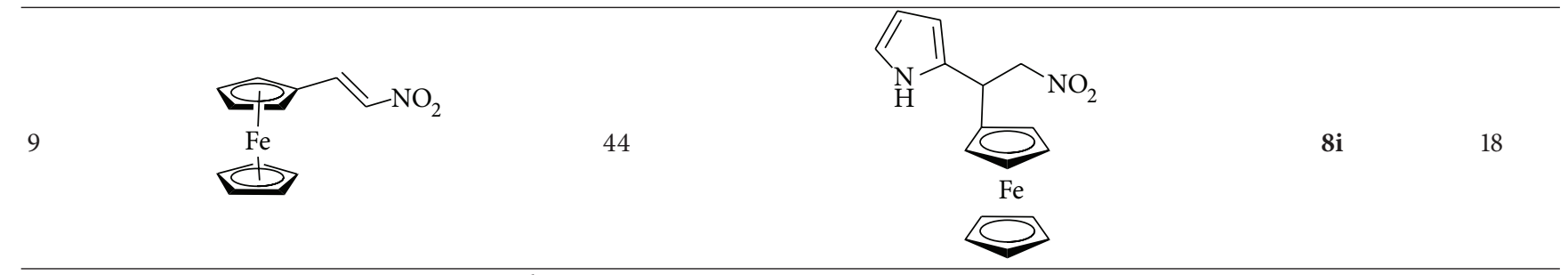

${ }^{a}$ The reactions were performed on $0.86 \mathrm{mmol} \mathrm{scale}$; ${ }^{b}$ the isolated yield after column purification.

$\left(\mathrm{CHCH}_{2}\right), 79.4\left(\mathrm{CHCH}_{2}\right), 105.7\left(\mathrm{PyC}_{2}\right), 108.7\left(\mathrm{PyC}_{3}\right), 118.2$ $\left(\mathrm{PyC}_{4}\right), 127.9$ (2C, $\left.\mathrm{PhC}_{2}\right), 129.2\left(\mathrm{PyC}_{1}\right), 130$ (2C, $\left.\mathrm{PhC}_{3}\right), 134.9$ $\left(\mathrm{PhC}_{4}\right), 138.0\left(\mathrm{PhC}_{1}\right)$; [Anal. Calcd. for $\mathrm{C}_{13} \mathrm{H}_{14} \mathrm{~N}_{2} \mathrm{O}_{2}: \mathrm{C}, 67.81$; $\mathrm{H}, 6.13$; N, 12.17; found: C, 68.07; H, 5.95; N, 11.91]; LC/MS (ESI): $\mathrm{M}^{+}$, found 230.06, $\mathrm{C}_{13} \mathrm{H}_{14} \mathrm{~N}_{2} \mathrm{O}_{2}$ requires 230.11; (minor region-isomer 9b): $\mathrm{IR}(\mathrm{KBr}): 3388,1514,1377,717,519 \mathrm{~cm}^{-1}$; ${ }^{1} \mathrm{H}-\mathrm{NMR}\left(\mathrm{CDCl}_{3}, 400 \mathrm{MHz}\right) \delta 2.25\left(\mathrm{~s}, 1 \mathrm{H}, \mathrm{CH}_{3}\right), 4.54-4.79$ $\left(\mathrm{m}, 4 \mathrm{H}, \mathrm{CHCH}_{2}\right), 4.79-4.82\left(\mathrm{~m}, 2 \mathrm{H}, \mathrm{CHCH}_{2}\right), 5.86-5.94(\mathrm{~m}$, 2H, ArH), 6.89-6.98 (m, 4H, ArH), 6.98-7.09 (m, 4H, ArH), 7.45 (s, $1 \mathrm{H}, \mathrm{NH}$ of pyrrole); ${ }^{13} \mathrm{C}-\mathrm{NMR}\left(\mathrm{CDCl}_{3}, 100 \mathrm{MHz}\right)$ : $\delta 21.1\left(\mathrm{CH}_{3}\right), 42.6\left(\mathrm{CHCH}_{2}\right), 79.3\left(\mathrm{CHCH}_{2}\right), 106.0\left(\mathrm{PyC}_{2}\right)$, $106.4\left(\mathrm{PyC}_{2}\right), 127.7\left(\mathrm{PhC}_{2}\right), 129.9\left(\mathrm{PyC}_{1}\right), 129.9\left(\mathrm{PhC}_{3}\right), 134.7$ $\left(\mathrm{PhC}_{4}\right), 137.9\left(\mathrm{PhC}_{1}\right)$; [Anal. Calcd. for $\mathrm{C}_{22} \mathrm{H}_{23} \mathrm{~N}_{3} \mathrm{O}_{4}$ : C, 67.16; $\mathrm{H}, 5.89$; N, 10.68; found: C, 66.86; H, 6.13; N, 10.94]; LC/MS (ESI): $\mathrm{M}^{+}$, found 393.19, $\mathrm{C}_{22} \mathrm{H}_{23} \mathrm{~N}_{3} \mathrm{O}_{4}$ requires 393.17.

2.3.3. 2-(1-(4-Methoxyphenyl)-2-nitroethyl)-1H-pyrrole (Table 3, Entry 3, 8c) and 2,5-Bis(1-(4-methoxy-phenyl)-2-nitroethyl)1H-pyrrole (Table 3, Entry 3, 9c). Pyrrole 5 (100 mg, $0.86 \mathrm{mmol})$ and $\beta$-nitroolefin $2 \mathrm{c}(154 \mathrm{mg}, 0.86 \mathrm{mmol})$ in isopropanol $(7 \mathrm{~mL})$ were reacted in the presence of Feist's acid (1) (12 mg, $0.168 \mathrm{mmol}, 10 \mathrm{~mol} \%$ ) according to GP2. The products were isolated by column chromatography on silica (EtOAc/hexane $0.5: 9.5$ ) yielded as oily liquid $\mathbf{8 c}$ (major region-isomer) (177 $\mathrm{mg}, 0.72 \mathrm{mmol}, 84 \%$ ) and 9c (minor region-isomer) as yellow oil $(50 \mathrm{mg}, 0.12 \mathrm{mmol}$, 14\%). Major region-isomer 8c: IR (KBr): 3388, 1542, 1378, $716,584 \mathrm{~cm}^{-1}$; ${ }^{1} \mathrm{H}-\mathrm{NMR}\left(\mathrm{CDCl}_{3}, 400 \mathrm{MHz}\right) \delta 3.78(\mathrm{~s}, 1 \mathrm{H}$, $\left.\mathrm{OCH}_{3}\right), 4.69-4.80\left(\mathrm{~m}, 1 \mathrm{H}, \mathrm{CHCH}_{2(\mathrm{a})}\right), 4.80-4.89(\mathrm{~m}, 1 \mathrm{H}$, $\left.\mathrm{CHCH}_{2(\mathrm{~b})}\right), 4.90-5.01$ (m, 1H, $\left.\mathrm{CHCH}_{2}\right), 6.06$ (s, 1H, ArH), 6.15-6.22 (m, 1H, ArH), 6.67 (s, 1H. ArH), $6.86(\mathrm{~d}, 2 \mathrm{H}$, $J=8.8 \mathrm{~Hz}, \operatorname{ArH}), 7.13(\mathrm{~d}, 2 \mathrm{H}, J=8.0 \mathrm{~Hz}, \operatorname{ArH}), 7.87(\mathrm{~s}$, $1 \mathrm{H}, \mathrm{NH}$ of pyrrole); ${ }^{13} \mathrm{C}-\mathrm{NMR}\left(\mathrm{CDCl}_{3}, 100 \mathrm{MHz}\right): \delta 42.3$ $\left(\mathrm{CHCH}_{2}\right), 55.4\left(\mathrm{OCH}_{3}\right), 79.5\left(\mathrm{CHCH}_{2}\right), 105.6\left(\mathrm{PyC}_{2}\right)$, $108.7\left(\mathrm{PyC}_{3}\right), 114.6\left(2 \mathrm{C}, \mathrm{PhC}_{3}\right), 118.2\left(\mathrm{PyC}_{4}\right), 129.1(2 \mathrm{C}$, $\left.\mathrm{PhC}_{2}\right), 129.4\left(\mathrm{PyC}_{1}\right), 130\left(\mathrm{PhC}_{4}\right), 159.4\left(\mathrm{PhC}_{1}\right) ;$ [Anal. Calcd. for $\mathrm{C}_{13} \mathrm{H}_{14} \mathrm{~N}_{2} \mathrm{O}_{3}$ : C, 63.40; $\mathrm{H}, 5.73 ; \mathrm{N}, 11.38$; found: $\mathrm{C}$, 63.62; H, 5.58; N, 11.19]; LC/MS (ESI): $\mathrm{M}^{+}$, found 246.14, $\mathrm{C}_{13} \mathrm{H}_{14} \mathrm{~N}_{2} \mathrm{O}_{3}$ requires 246.10; minor region-isomer 9c: IR (KBr): 3388, 1514, 1377, 717, $519 \mathrm{~cm}^{-1} ;{ }^{1} \mathrm{H}-\mathrm{NMR}\left(\mathrm{CDCl}_{3}\right.$, $400 \mathrm{MHz}) \delta 3.76\left(\mathrm{~s}, 1 \mathrm{H}, \mathrm{CH}_{3}\right), 4.60-4.78\left(\mathrm{~m}, 4 \mathrm{H}, \mathrm{CHCH}_{2}\right)$, 4.78-4.93 (m, 2H, $\left.\mathrm{CHCH}_{2}\right), 5.92-6.01$ (m, 2H, ArH), 6.80-6.84 (m, 4H, ArH), 6.01-7.12 (m, 4H, ArH), 7.54 (s, $1 \mathrm{H}, \mathrm{NH}$ of pyrrole); ${ }^{13} \mathrm{C}-\mathrm{NMR}\left(\mathrm{CDCl}_{3}, 100 \mathrm{MHz}\right): \delta 41.2$ $\left(\mathrm{CHCH}_{2}\right), 55.4\left(\mathrm{CH}_{3}\right), 79.4\left(\mathrm{CHCH}_{2}\right), 105.9\left(\mathrm{PyC}_{2}\right), 106.3$ $\left(\mathrm{PyC}_{2}\right), 114.6\left(\mathrm{PhC}_{3}\right), 128.96\left(\mathrm{PhC}_{2}\right), 129.02\left(\mathrm{PyC}_{1}\right), 129.8$ $\left(\mathrm{PhC}_{1}\right), 159.4\left(\mathrm{PhC}_{4}\right)$; [Anal. Calcd. for $\mathrm{C}_{22} \mathrm{H}_{23} \mathrm{~N}_{3} \mathrm{O}_{6}$ : C,
62.11; H, 5.45; N, 9.88; found: C, 62.27; H, 5.39; N, 10.13]; LC/MS (ESI): $\mathrm{M}^{+}$, found 425.13, $\mathrm{C}_{22} \mathrm{H}_{23} \mathrm{~N}_{3} \mathrm{O}_{6}$ requires 425.16 .

2.3.4. 2-(1-(4-Chlorophenyl)-2-nitroethyl)-1H-pyrrole (Table 3, Entry 4, 8d) and 2,5-Bis(1-(4-chlorophenyl)-2-nitroethyl)-1Hpyrrole (Table 3, Entry 4, 9d). Pyrrole 5 (100 mg, $0.86 \mathrm{mmol}$ ) and $\beta$-nitroolefin $2 \mathbf{d}$ ( $157 \mathrm{mg}, 0.86 \mathrm{mmol})$ in dry ethanol $(7 \mathrm{~mL})$ were reacted in the presence of Feist's acid (1) $(12 \mathrm{mg}$, $0.168 \mathrm{mmol}, 10 \mathrm{~mol} \%$ ) according to GP2. The products were isolated by column chromatography on silica (EtOAc/hexane $0.5: 9.5)$ yielded white solid $\mathbf{8 d}$ (major region-isomer) (140 $\mathrm{mg}, 0.56 \mathrm{mmol}, 65 \%$ ) and $\mathbf{9 d}$ (minor region-isomer) as yellow oil (40 mg, $0.09 \mathrm{mmol}, 11 \%$ ). Major region-isomer $\mathbf{8 d}$ : IR (KBr): 3379, 1740, 1545, 1377, 1092, 725, 512, $452 \mathrm{~cm}^{-1} ;{ }^{1} \mathrm{H}-$ $\mathrm{NMR}\left(\mathrm{CDCl}_{3}, 400 \mathrm{MHz}\right) \delta 4.66-4.83\left(\mathrm{~m}, 2 \mathrm{H}, \mathrm{CHCH}_{2(\mathrm{a})}\right)$, 4.83-4.91 (m, 2H, $\left.\mathrm{CHCH}_{2(\mathrm{~b})}\right), 4.91-5.06\left(\mathrm{~m}, 1 \mathrm{H}, \mathrm{CHCH}_{2}\right)$, 6.07 (s, 1H, ArH), 6.12-6.21 (m, 1H, ArH), 6.70 (s, 1H. ArH), $7.17(\mathrm{~d}, 2 \mathrm{H}, J=6.84 \mathrm{~Hz}, \operatorname{ArH}), 7.33(\mathrm{~d}, 2 \mathrm{H}, J=6.84$, $\mathrm{Hz}, \mathrm{ArH}), 7.84$ (s, $1 \mathrm{H}, \mathrm{NH}$ of pyrrole); ${ }^{13} \mathrm{C}-\mathrm{NMR}\left(\mathrm{CDCl}_{3}\right.$, $100 \mathrm{MHz}): \delta 42.4\left(\mathrm{CHCH}_{2}\right), 79.0\left(\mathrm{CHCH}_{2}\right), 106.1\left(\mathrm{PyC}_{2}\right)$, $108.9\left(\mathrm{PyC}_{3}\right), 118.6\left(\mathrm{PyC}_{4}\right), 128.4\left(\mathrm{PhC}_{4}\right), 129.4\left(2 \mathrm{C}, \mathrm{PhC}_{2}\right)$, 129.5 (2C, $\left.\mathrm{PhC}_{3}\right), 134.2\left(\mathrm{PyC}_{1}\right), 136.6\left(\mathrm{PhC}_{1}\right)$; [Anal. Calcd. for $\mathrm{C}_{12} \mathrm{H}_{11} \mathrm{ClN}_{2} \mathrm{O}_{2}$ : C, 57.49; H, 4.42; N, 11.17; found: C, 57.36; $\mathrm{H}$, 4.53; N, 11.01]; LC/MS (ESI): $\mathrm{M}^{+} \&[\mathrm{M}+2]^{+}$, found $250.08 \&$ 252.03, $\mathrm{C}_{12} \mathrm{H}_{11} \mathrm{ClN}_{2} \mathrm{O}_{2}$ requires 250.05; minor region-isomer 9d: IR (KBr): 3413, 1549, 1490, 1375, 1091, 726, $512 \mathrm{~cm}^{-1} ;{ }^{1} \mathrm{H}-$ $\operatorname{NMR}\left(\mathrm{CDCl}_{3}, 400 \mathrm{MHz}\right) \delta 4.61-4.82\left(\mathrm{~m}, 4 \mathrm{H}, \mathrm{CHCH}_{2}\right), 4.82-$ $5.03\left(\mathrm{~m}, 2 \mathrm{H}, \mathrm{CHCH}_{2}\right), 5.93-6.02(\mathrm{~m}, 2 \mathrm{H}, \mathrm{ArH}), 7.01-7.20$ (m, 4H, ArH), 7.20-7.39 (m, 4H, ArH), 7.53 (s, 1H, NH of pyrrole); ${ }^{13} \mathrm{C}-\mathrm{NMR}\left(\mathrm{CDCl}_{3}, 100 \mathrm{MHz}\right): \delta 42.2\left(\mathrm{CHCH}_{2}\right), 78.9$ $\left(\mathrm{CHCH}_{2}\right), 106.5\left(\mathrm{PyC}_{2}\right), 106.9\left(\mathrm{PyC}_{2}\right), 129.2\left(\mathrm{PhC}_{3}\right), 129.3$ $\left(\mathrm{PhC}_{2}\right), 129.52\left(\mathrm{PyC}_{1}\right), 134.3\left(\mathrm{PhC}_{4}\right), 136.2\left(\mathrm{PhC}_{1}\right)$; [Anal. Calcd. for $\mathrm{C}_{20} \mathrm{H}_{17} \mathrm{Cl}_{2} \mathrm{~N}_{3} \mathrm{O}_{4}$ : C, 55.31; H, 3.95; N, 9.68; found: C, 55.19; H, 4.14; N, 9.43]; LC/MS (ESI): $\mathrm{M}^{+} \&[\mathrm{M}+2]^{+}$, found $433.11 \& 435.15, \mathrm{C}_{20} \mathrm{H}_{17} \mathrm{Cl}_{2} \mathrm{~N}_{3} \mathrm{O}_{4}$ requires 433.05.

2.3.5. 2-(1-(4-Bromophenyl)-2-nitroethyl)-1H-pyrrole (Table 3, Entry 5, 8e). Pyrrole 5 (100 mg, $0.86 \mathrm{mmol})$ and $\beta$-nitroolefin $2 \mathbf{e}(195 \mathrm{mg}, 0.86 \mathrm{mmol})$ in isopropanol $(7 \mathrm{~mL})$ were reacted in the presence of Feist's acid (1) (12 mg, $0.168 \mathrm{mmol}, 10 \mathrm{~mol} \%$ ) according to GP2. The product was isolated by column chromatography on silica (EtOAc/hexane $0.5: 9.5$ ) yielded as white solid 8e (120 mg, $0.41 \mathrm{mmol}, 47 \%)$. IR (KBr): 3376, 1543, $1377,1007,725,510 \mathrm{~cm}^{-1} ;{ }^{1} \mathrm{H}-\mathrm{NMR}\left(\mathrm{CDCl}_{3}, 400 \mathrm{MHz}\right) \delta 4.71-$ $4.81\left(\mathrm{~m}, 1 \mathrm{H}, \mathrm{CHCH}_{2(\mathrm{a})}\right), 4.81-4.90\left(\mathrm{~m}, 1 \mathrm{H}, \mathrm{CHCH}_{2(\mathrm{~b})}\right), 4.90-$ $5.02\left(\mathrm{~m}, 2 \mathrm{H}, \mathrm{CHCH}_{2}\right), 6.07$ (s, 1H, ArH), 6.12-6.22 (m, 1H, 
ArH), 6.70 (s, 1H. ArH), 7.11 (d, 2H, $J=8.8 \mathrm{~Hz}, \operatorname{ArH}), 7.48$ (d, $2 \mathrm{H}, J=8.8, \mathrm{~Hz}, \mathrm{ArH}), 7.86$ (s, $1 \mathrm{H}, \mathrm{NH}$ of pyrrole); ${ }^{13} \mathrm{C}$ NMR $\left(\mathrm{CDCl}_{3}, 100 \mathrm{MHz}\right): \delta 42.5\left(\mathrm{CHCH}_{2}\right), 79.0\left(\mathrm{CHCH}_{2}\right)$, $106.1\left(\mathrm{PyC}_{2}\right), 108.9\left(\mathrm{PyC}_{3}\right), 118.6\left(\mathrm{PyC}_{4}\right), 122.3\left(\mathrm{PhC}_{4}\right), 128.3$ $\left(\mathrm{PyC}_{1}\right), 129.7$ (2C, $\left.\mathrm{PhC}_{2}\right), 132.4\left(\mathrm{PhC}_{3}\right), 137.1\left(\mathrm{PhC}_{1}\right)$; [Anal. Calcd. for $\mathrm{C}_{12} \mathrm{H}_{11} \mathrm{BrN}_{2} \mathrm{O}_{2}$ : C, 48.84; $\mathrm{H}, 3.76$; N, 9.49; found: C, 49.07; H, 3.89; N, 9.35]; LC/MS (ESI): $\mathrm{M}^{+} \&[\mathrm{M}+2]^{+}$, found $294.01 \& 296.05, \mathrm{C}_{12} \mathrm{H}_{11} \mathrm{BrN}_{2} \mathrm{O}_{2}$ requires 294.0.

2.3.6. 2-(2-Nitro-1-(4-nitrophenyl) ethyl)-1H-pyrrole (Table 3, Entry 6, 8f). Pyrrole 5 (100 mg, $0.8 \mathrm{mmol})$ and $\beta$-nitroolefin $2 f(167 \mathrm{mg}, 0.86 \mathrm{mmol})$ in isopropanol $(7 \mathrm{~mL})$ were reacted in the presence of Feist's acid (1) (12 mg, $0.168 \mathrm{mmol}, 10 \mathrm{~mol} \%)$ according to GP2. The product was isolated by column chromatography on silica (EtOAc/hexane $0.5: 9.5$ ) yielded as oily liquid $\mathbf{8 f}$ (224 mg, $0.86 \mathrm{mmol}, 99.8 \%)$. IR (KBr): $3421,1551,1520,1373,1349,784,717 \mathrm{~cm}^{-1} ;{ }^{1} \mathrm{H}-\mathrm{NMR}\left(\mathrm{CDCl}_{3}\right.$, $400 \mathrm{MHz}) \delta$ 4.93-5.05 (m, 2H, $\left.\mathrm{CHCH}_{2}\right), 5.51-5.57(\mathrm{~m}, 1 \mathrm{H}$, $\mathrm{CHCH}_{2}$ ), 6.12-6.19 (m, 2H, ArH), 6.73 (s, 1H. ArH), 7.31-7.48 (m, 2H, ArH), 7.48-7.61 (m, 1H, ArH), 7.81-7.93 (m, 1H, ArH), 8.45 (s, $1 \mathrm{H}, \mathrm{NH}$ of pyrrole); ${ }^{13} \mathrm{C}-\mathrm{NMR}\left(\mathrm{CDCl}_{3}, 100 \mathrm{MHz}\right): \delta$ $36.9\left(\mathrm{CHCH}_{2}\right), 79.8\left(\mathrm{CHCH}_{2}\right), 105.6\left(\mathrm{PyC}_{2}\right), 108.8\left(\mathrm{PyC}_{3}\right)$, $118.9\left(\mathrm{PyC}_{4}\right), 127.8\left(\mathrm{PyC}_{3}\right), 128.8\left(2 \mathrm{C}, \mathrm{PhC}_{2}\right), 129.4\left(\mathrm{PyC}_{1}\right)$, $133.7\left(\mathrm{PhC}_{4}\right), 149.6\left(\mathrm{PhC}_{1}\right)$; [Anal. Calcd. for $\mathrm{C}_{12} \mathrm{H}_{11} \mathrm{~N}_{3} \mathrm{O}_{4}$ : C, 55.17; H, 4.24; N, 16.09; found: C, 55.36; H, 4.19; N, 15.93]; LC/MS (ESI): $\mathrm{M}^{+}$, found 261.15, $\mathrm{C}_{12} \mathrm{H}_{11} \mathrm{~N}_{3} \mathrm{O}_{4}$ requires 261.07.

2.3.7.2-(1-(2,4-Dichlorophenyl)-2-nitroethyl)-1H-pyrrole (Table 3, Entry 7, 8g). Pyrrole 5 (100 mg, $0.86 \mathrm{mmol})$ and $\beta$ nitroolefin $2 \mathrm{~g}(187 \mathrm{mg}, 0.86 \mathrm{mmol})$ in isopropanol $(7 \mathrm{~mL})$ were reacted in the presence of Feist's acid (1) (12 mg, $0.168 \mathrm{mmol}, 10 \mathrm{~mol} \%$ ) according to GP2. The product was isolated by column chromatography on silica (EtOAc/hexane $1: 9)$ yielded as oily liquid $\mathbf{8 g}$ (245 $\mathrm{mg}, 0.86 \mathrm{mmol}, 99.9 \%)$. IR (KBr): 3417, 1550, 1469, 1376, 1099, 797, $726 \mathrm{~cm}^{-1} ;{ }^{1} \mathrm{H}-\mathrm{NMR}$ $\left(\mathrm{CDCl}_{3}, 400 \mathrm{MHz}\right) \delta 4.71-4.98\left(\mathrm{~m}, 2 \mathrm{H}, \mathrm{CHCH}_{2}\right), 5.32-5.49$ $\left(\mathrm{m}, 1 \mathrm{H}, \mathrm{CHCH}_{2}\right), 6.06-6.23(\mathrm{~m}, 2 \mathrm{H}, \mathrm{ArH}), 6.71$ (s, 1H, ArH), 6.94-7.10 (m, 1H, ArH), 7.10-7.29 (m, 1H, ArH), 7.31-7.58 (m, $1 \mathrm{H}, \mathrm{ArH}), 8.02$ (s, $1 \mathrm{H}, \mathrm{NH}$ of pyrrole); ${ }^{13} \mathrm{C}-\mathrm{NMR}\left(\mathrm{CDCl}_{3}\right.$, $100 \mathrm{MHz}): \delta 39.0\left(\mathrm{CHCH}_{2}\right), 79.9\left(\mathrm{CHCH}_{2}\right), 106.6\left(\mathrm{PyC}_{2}\right)$, $108.9\left(\mathrm{PyC}_{3}\right), 118.7\left(\mathrm{PyC}_{4}\right), 127.4\left(\mathrm{PyC}_{1}\right), 128.0\left(\mathrm{PhC}_{6}\right), 130.0$ $\left(\mathrm{PhC}_{5}\right), 130.1\left(\mathrm{PhC}_{3}\right), 134.3-134.6\left(\mathrm{PhC}_{1}, \mathrm{PhC}_{2} \& \mathrm{PhC}_{4}\right)$; [Anal. Calcd. for $\mathrm{C}_{12} \mathrm{H}_{10} \mathrm{Cl}_{2} \mathrm{~N}_{2} \mathrm{O}_{2}$ : C, 50.55; H, 3.54; N, 9.82; found: C, 50.33; H, 3.69; N, 10.07]; LC/MS (ESI): $\mathrm{M}^{+}$, found 284.05, $\mathrm{C}_{12} \mathrm{H}_{11} \mathrm{~N}_{3} \mathrm{O}_{4}$ requires 284.01.

2.3.8. 2-(1-(2,6-Dichlorophenyl)-2-nitroethyl)-1H-pyrrole (Table 3, Entry 8, 8h) and 2,5-Bis(1-(2,6-dichloro-phenyl)-2-nitroethyl)1H-pyrrole (Table 3, Entry 8, 9h). Pyrrole 5 (100 mg, $0.86 \mathrm{mmol})$ and $\beta$-nitrostyrene $2 \mathbf{h}(187 \mathrm{mg}, 0.86 \mathrm{mmol})$ in isopropanol $(7 \mathrm{~mL})$ were reacted in the presence of Feist's acid (1) (12 mg, $0.168 \mathrm{mmol}, 10 \mathrm{~mol} \%$ ) according to GP2. The products were isolated by column chromatography on silica (EtOAc/hexane $0.5: 9.5$ ) yielded yellow oil $\mathbf{8 h}$ (major region-isomer) (220 $\mathrm{mg}, 0.77 \mathrm{mmol}, 90 \%$ ) and $\mathbf{9 h}$ (minor region-isomer) as yellow oil (40 mg, $0.09 \mathrm{mmol}, 9 \%)$. Major region-isomer 8h: IR (KBr): 3439, 1550, 1430, 1374, 775, 720, $536 \mathrm{~cm}^{-1} ;{ }^{1} \mathrm{H}-\mathrm{NMR}\left(\mathrm{CDCl}_{3}, 400 \mathrm{MHz}\right) \delta 5.21-5.30(\mathrm{~m}, 1 \mathrm{H}$,
$\left.\mathrm{CHCH}_{2(\mathrm{a})}\right), 5.30-4.42\left(\mathrm{~m}, 1 \mathrm{H}, \mathrm{CHCH}_{2(\mathrm{~b})}\right), 5.90-6.01$ (m, $\left.1 \mathrm{H}, \mathrm{CHCH}_{2}\right), 6.09$ (s, 1H, ArH), 6.12-6.28 (m, 1H, ArH), 6.66-6.75 (m, 1H. ArH), 7.12-7.24 (m, 1H, ArH), 7.28-7.48 (m, $2 \mathrm{H}, \mathrm{ArH}), 8.06$ (s, $1 \mathrm{H}, \mathrm{NH}$ of pyrrole); ${ }^{13} \mathrm{C}-\mathrm{NMR}\left(\mathrm{CDCl}_{3}\right.$, $100 \mathrm{MHz}): \delta 39.0\left(\mathrm{CHCH}_{2}\right), 79.5\left(\mathrm{CHCH}_{2}\right), 106.8\left(\mathrm{PyC}_{2}\right)$, $108.7\left(\mathrm{PyC}_{3}\right), 118.1\left(\mathrm{PyC}_{4}\right), 124.4\left(2 \mathrm{C}, \mathrm{PhC}_{3}\right), 126.8\left(\mathrm{PhC}_{4}\right)$, $129.8\left(2 \mathrm{C}, \mathrm{PhC}_{2}\right), 133.8\left(\mathrm{PyC}_{1}\right), 142.8\left(\mathrm{PhC}_{1}\right)$; [Anal. Calcd. for $\mathrm{C}_{12} \mathrm{H}_{10} \mathrm{Cl}_{2} \mathrm{~N}_{2} \mathrm{O}_{2}$ : C, 50.55; $\mathrm{H}, 3.54 ; \mathrm{N}, 9.82$; found: $\mathrm{C}$, 50.45; H, 3.43; N, 9.74]; LC/MS (ESI): $\mathrm{M}^{+} \&[\mathrm{M}+2]^{+}$, found $284.05 \& 286.11, \mathrm{C}_{12} \mathrm{H}_{10} \mathrm{Cl}_{2} \mathrm{~N}_{2} \mathrm{O}_{2}$ requires 284.01; minor region-isomer 9h: IR (KBr): 3437, 1741, 1551, 1431, 1372, 1210, $773,532 \mathrm{~cm}^{-1} ;{ }^{1} \mathrm{H}-\mathrm{NMR}\left(\mathrm{CDCl}_{3}, 400 \mathrm{MHz}\right) \delta 5.14-5.32(\mathrm{~m}$, $\left.4 \mathrm{H}, \mathrm{CHCH}_{2}\right), 5.83\left(\mathrm{t}, J=6.6 \mathrm{~Hz}, 2 \mathrm{H}, \mathrm{CHCH}_{2}\right), 5.93-6.02(\mathrm{~m}$, 2H, ArH), 7.12-7.23 (m, 2h, ArH), 7.26-7.42 (m, 4 h, ArH), 8.16 (s, $1 \mathrm{H}, \mathrm{NH}$ of pyrrole); ${ }^{13} \mathrm{C}-\mathrm{NMR}\left(\mathrm{CDCl}_{3}, 100 \mathrm{MHz}\right): \delta$ $42.2\left(\mathrm{CHCH}_{2}\right), 78.9\left(\mathrm{CHCH}_{2}\right), 106.5\left(\mathrm{PyC}_{2}\right), 106.9\left(\mathrm{PyC}_{2}\right)$, $129.2\left(\mathrm{PhC}_{3}\right), 129.3\left(\mathrm{PhC}_{2}\right), 129.52\left(\mathrm{PyC}_{1}\right), 134.3\left(\mathrm{PhC}_{4}\right), 136.2$ $\left(\mathrm{PhC}_{1}\right.$ ); [Anal. Calcd. for $\mathrm{C}_{20} \mathrm{H}_{15} \mathrm{Cl}_{4} \mathrm{~N}_{3} \mathrm{O}_{4}$ : C, 47.74; $\mathrm{H}, 3.0$; N, 8.35; found: C, 48.09; H, 3.11; N, 8.51]; LC/MS (ESI): $\mathrm{M}^{+} \&$ $[\mathrm{M}+2]^{+}$, found 501.03 and $503.06, \mathrm{C}_{20} \mathrm{H}_{15} \mathrm{Cl}_{4} \mathrm{~N}_{3} \mathrm{O}_{4}$ requires 500.98 .

2.3.9. 2-(1-Ferrocenyl)-2-nitroethyl)-1H-pyrrole (Table 3, Entry 9, 8i). Pyrrole 5 (100 mg, $0.86 \mathrm{mmol}$ ) and $\beta$-nitroolefin $\mathbf{2 g}$ $(167 \mathrm{mg}, 0.86 \mathrm{mmol})$ in dry ethanol $(7 \mathrm{~mL})$ were reacted in the presence of Feist's acid (1) (12 mg, $0.168 \mathrm{mmol}, 10 \mathrm{~mol} \%$ ) according to GP2. The product was isolated by column chromatography on silica (EtOAc/hexane 0.5:9.5) dark red solid 8g (51 mg, 18\%). IR (KBr): 3358, 1740, 1551, 1410, 1251, $1219,1044,728,488 \mathrm{~cm}^{-1} ;{ }^{1} \mathrm{H}-\mathrm{NMR}\left(\mathrm{CDCl}_{3}, 400 \mathrm{MHz}\right) \delta 4.13$ (s, $1 \mathrm{H}$, protons of $\mathrm{C} p$ ), 4.23 (s, $5 \mathrm{H}$, protons of $\mathrm{Cp}$ ), 4.30-4.39 $(\mathrm{m}, 2 \mathrm{H}$, protons of $\mathrm{Cp}), 4.64(\mathrm{~s}, 2 \mathrm{H}, \mathrm{m}, 2 \mathrm{H}$, protons of $\mathrm{C} p)$, 5.10-5.21 (m, 1H, $\left.\mathrm{CHCH}_{2}\right), 5.21-5.40\left(\mathrm{~m}, 2 \mathrm{H}, \mathrm{CHCH}_{2}\right), 6.26-$ 6.36 (m, 1H, ArH), 6.81-6.90 (m, 1H, ArH), 7.18 (m, 1H, ArH), 7.79 (s, 1H, NH of pyrrole); ${ }^{13} \mathrm{C}-\mathrm{NMR}\left(\mathrm{CDCl}_{3}, 100 \mathrm{MHz}\right): \delta$ 29.8, 69.1, 70.0, 70.6, 72.3, 84.0, 111.6, 123.0, 125.2, 126.6; [Anal. Calcd. for $\mathrm{C}_{16} \mathrm{H}_{16} \mathrm{FeN}_{2} \mathrm{O}_{2}$ : C, 59.28; $\mathrm{H}, 4.98 ; \mathrm{N}, 8.64$; found: C, 59.17; H, 5.07; N, 8.49]; LC/MS (ESI): $\mathrm{M}^{+}$, found 324.12, $\mathrm{C}_{16} \mathrm{H}_{16} \mathrm{FeN}_{2} \mathrm{O}_{2}$ requires 324.16.

\section{Result and Discussion}

At the outset, synthetic strategies adopted in our work, for the preparation of Feist's acid (FA) (1) as hydrogen bond donor catalyst, has been outlined in Scheme 1. Feist's acid is commercially available but highly expensive therefore, it was prepared in our laboratory from a very cheap and readily available material ethyl acetoacetate with an overall yield of $19 \%$ in three steps, using the well-known method reported by Al-Majid et al. [50].

Initially, to evaluate the catalytic activity of Feist's acid for the Michel addition reaction, indole $\mathbf{4}$ and $\beta$-nitrostyrene $\mathbf{2 a}$ have been chosen as model substrates. 5-20 mol\% of FA (1) were used, aiming at screening the optimal conditions, and the results are summarized in Table 1.

By screening different solvents such as methanol, ethanol, isopropanol ( $\left.{ }^{\mathrm{i}} \mathrm{PrOH}\right)$, toluene, benzene, xylene, acetonitrile, tetrahydrofuran (THF), and dichloromethane $\left(\mathrm{CH}_{2} \mathrm{Cl}_{2}\right)$, using $10 \mathrm{~mol} \%$ of Feist's acid, we found that most of the 

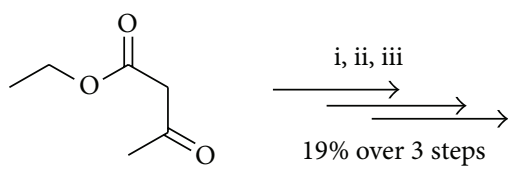

Ethyl acetoacetate

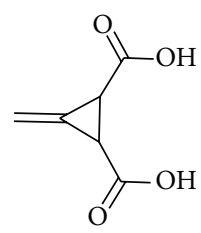

Feist's acid (1)
Reagents and conditions:

(i) $\mathrm{Dry} \mathrm{HCl}$ (g) 14 days in a dark place

(ii) $\mathrm{Br}_{2} \mathrm{CHCl}_{3} 24 \mathrm{~h} \mathrm{RT}$

(iii) $7 \mathrm{M} \mathrm{KOH} 105^{\circ} \mathrm{C} 1 \mathrm{~h}$

Scheme 1: Feist's acid synthesis.

solvents produced good to excellent yields (86-96\%) for the Michael addition reaction of indole 4 to $\beta$-nitrostyrene $\mathbf{2 a}$, affording the corresponding product $7 \mathbf{a}$ at $50-60^{\circ} \mathrm{C}$ temperature within $24-42 \mathrm{~h}$, (Table 1, entries 5-10,14), while in case of $\mathrm{ACN}$ and $\mathrm{MeOH}$, moderate yields were observed (49\% and $62 \%$ ), respectively (Table 1 , entries 1,4 ). But in case of other solvents like $\mathrm{CH}_{2} \mathrm{Cl}_{2}$ and THF, no products were formed (Table 1, entries 2,3). Without using Feist's acid as catalyst, the reaction was carried out in ethanol at room temperature as well as at $60^{\circ} \mathrm{C}$ for $72 \mathrm{~h}$; no product formation was observed at all (Table 1, entries 11, 12). But with the use $5 \mathrm{~mol} \%$ catalyst in ethanol at $50^{\circ} \mathrm{C}$ for $60 \mathrm{~h}, 59 \%$ yield was observed (Table 1 , entry 13). The yields of product were improved remarkably from $59 \%$ to $98 \%$ by increasing the loading of catalyst from 5 to $20 \mathrm{~mol} \%$ in ethanol (Table 1, entries 13, 14). It is noteworthy to mention that solvents like DMF and $\mathrm{AcOH}$ with $10 \%$ catalyst produced very good results in $42 \mathrm{~h}$ and $18 \mathrm{~h}$ accordingly (Table 1 , entries 5,7 ). But due to the workup problem these solvents cause they have been discarded from further reaction optimization. Xylene, toluene, and benzene also have been discarded for their high boiling point as compared to ethanol. The optimized procedure for Michael addition of indole to nitroolefin was found to be as follows: the mixture of indole $4(0.43 \mathrm{mmol}), \beta$-nitrostyrene $2 a(0.43 \mathrm{mmol})$, and FA (Feist's acid, $10 \mathrm{~mol} \%$ ) was heated at $50^{\circ} \mathrm{C}$ in ethanol for $42 \mathrm{~h}$ according to general procedure GP1.

To illustrate the generality of this Michael addition reaction of indole 4 , with various nitroolefins $2(a-i)$, sixteen examples were carried out catalyzed by Feist's acid with $10 \mathrm{~mol} \%$ and $20 \mathrm{~mol} \%$ in ethanol at $50^{\circ} \mathrm{C}$ temperature and, the results are shown in Table 2.

The exclusive 3 -substituted indole derivatives $7 \mathbf{a}$-i were obtained in good to excellent yields (46-97\%) with the use of $10 \mathrm{~mol} \%$ of catalyst at $50^{\circ} \mathrm{C}$ temperature in 48 hours (Table 2 , entries 1-13). The reaction of indole (4) with nitroolefins 2(e-h) bearing 4-bromophenyl, 2,4-dichlorophenyl, 2,6dichlorophenyl, and 4-nitrophenyl produced the corresponding Michael adducts $7(\mathbf{e}-\mathbf{h})$ with poor to moderate yield $(49 \%, 55 \%, 63 \%$, and $62 \%)$, respectively (Table 2 , entries 5,7 , 9 , and 11). The poor reactivity of these nitroolefins could be inferred due to their less solubility and bulkier environment. But the chemical yields were increased dramatically to $87 \%$, $70 \%, 97 \%$, and $75 \%$, respectively, when the loading of catalyst were increased from $10-20 \mathrm{~mol} \%$ and the reactions were run for 72 hours (Table 2, entries $6^{\mathrm{c}}, 8^{\mathrm{c}}, 10^{\mathrm{c}}, 12^{\mathrm{c}}$ ). In contrast, the nitroolefin bearing phenyl, tolyl, and 4-chlorophenyl, afforded the corresponding Michael products in excellent yield (97\%) (Table 2, entries 1, 2 and 4), while in the case of nitroolefin with 4-methoxyphenyl, the yield was slightly lower (84\%) (Table 2, entry 3), which was probably attributed to the steric effect of the 4-methoxyphenyl group.

On the basis of the above results obtained for Michael addition reaction of indole with the derivatives of $\beta$ nitrostyrenes, the reaction was extended to pyrrole, and it was found that Feist's acid (FA) can also efficiently catalyze the reaction of pyrrole with different $\beta$-nitroolefins in isopropanol, affording 2 -substituted pyrrole and in some cases both 2-substituted and 2,4-disubstituted pyrrole derivatives in good to excellent yields; the results are summarized in Table 3.

The Michael addition of pyrrole 5 to nitroolefins $2(\mathbf{a}-\mathbf{i})$ catalyzed by FA ( $10 \mathrm{~mol} \%)$ in isopropanol at $50^{\circ} \mathrm{C}$ for $20-50 \mathrm{~h}$ afforded 2-substituted pyrrole $\mathbf{8}(\mathbf{a}-\mathbf{i})$ as major region-isomer (Table 3, entries 1-9), with some 2,4-disubstituted pyrrole derivatives $\mathbf{9}(\mathbf{a}-\mathbf{d})$ and $\mathbf{9 h}$ (Table 3 , entries $1-4,8$ ), which also showed good regioselectivity of pyrrole at the 2-position. The reaction of pyrrole with nitroolefins bearing phenyl, tolyl, 4-methoxyphenyl, 4-chloroophenyl and, 24-dichlorophenyl $\mathbf{2}(\mathbf{a}-\mathbf{d})$ and $\mathbf{2 h}$ afforded major region-isomer 2-substituted pyrrole $\mathbf{8}(\mathbf{a}-\mathbf{d})$ and $\mathbf{8 h}(81 \%, 50 \%, 84 \%, 65 \%$, and 90\%) with some minor region-isomer 2,6-disubstituted pyrrole 9(a-d) and $9 \mathbf{h}(13 \%, 21 \%, 13 \%, 11 \%$, and $9 \%)$ in $50 \mathrm{~h}, 24 \mathrm{~h}$, $44 \mathrm{~h}, 20 \mathrm{~h}$, and $44 \mathrm{~h}$, respectively (Table 3 , entries $1-4,8$ ). But 2-substituted Michael adducts $8(\mathbf{e}-\mathbf{g})$ and $\mathbf{8 i}$ were formed exclusively with $48 \%, 99.8 \%, 99.9 \%$, and $18 \%$, when nitroolefins bearing 4-bromophenyl, 4-nitrophenyl, 2,4dichlorophenyl, and ferrocene groups reacted with pyrrole in $20 \mathrm{~h}$ and $44 \mathrm{~h}$, accordingly (Table 3, entries 5-7, 9). Obviously the best results were found in the case of nitroolefin $\mathbf{2} \mathbf{f}$ and $\mathbf{2 g}$ producing their corresponding adducts $\mathbf{8 f}$ and $\mathbf{8 g}$ with excellent yields 99.8 and $99.9 \%$, respectively (Table 3, entries 6,7 ). On the other hand, poor yields (8e and $8 \mathbf{i}$ with $48 \%$ and $18 \%$ ) were observed corresponding to the nitroolefins $2 \mathrm{e}$ and $2 \mathbf{i}$. The reason for this low yield could be attributed to the poor solubility of $2 \mathbf{e}$ and $2 \mathbf{i}$.

We reasoned that, the catalytic cycle would begin with activation of an electrophile, such as $\beta$-nitrostyrenes (2a-i), with Feist's acid (1) to afford intermediate 3 (Figure 1). Addition of nucleophiles ( 4 and $\mathbf{5}$ ), to the intermediate 3 give rise to another intermediate 6 followed by proton transfer and release of the catalyst (1), would complete the cycle and generate the desired products $7 \mathbf{a}-\mathbf{i}, \mathbf{8} \mathbf{a}-\mathbf{i}, \mathbf{9 a}-\mathbf{d}$ and $\mathbf{9 h}$ corresponding to the substrate $\mathbf{4}$ and $\mathbf{5}$ (Tables 2 and 3). All the analytical data are available the Supplementary Material available online at http//dox.doi.org/10.1155/2014/649197.

\section{General Procedure for Antimicrobial Activity}

Chemical compounds were individually tested against a panel of Gram-positive and Gram-negative bacterial pathogens. Antimicrobial tests were carried out by the agar well diffusion method using $100 \mathrm{~mL}$ of suspension containing $1 \times$ 


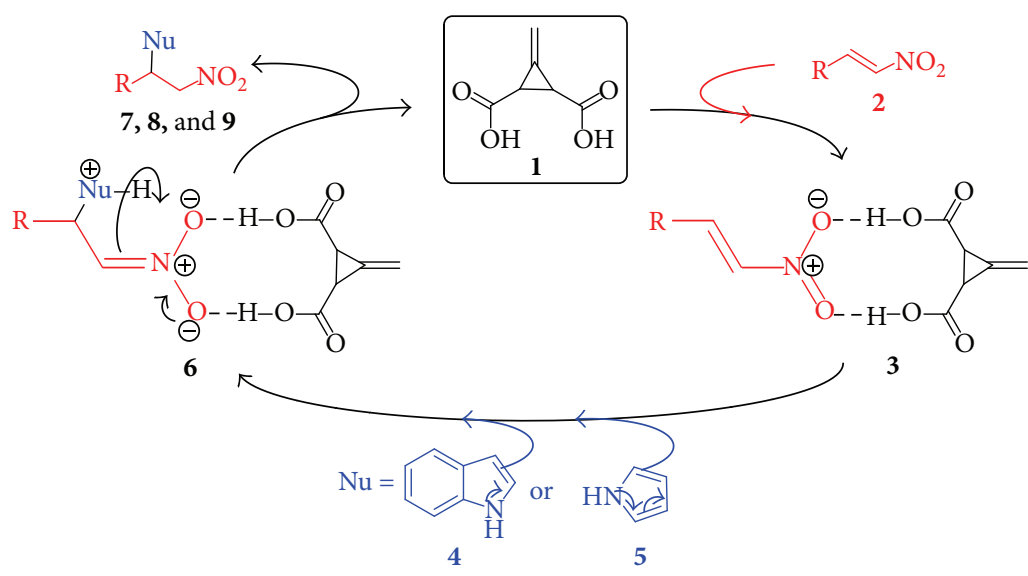

FIGURE 1: Activation of nitrostyrene via hydrogen bonding mechanism by Feist's acid catalysis.

TABLE 4: Antimicrobial activity of the newly synthesized compounds against the pathological strains based on well diffusion assay ${ }^{\mathrm{a}}$.

\begin{tabular}{|c|c|c|c|c|c|}
\hline \multirow{2}{*}{ Comp. no. } & \multicolumn{2}{|c|}{ Gram-positive bacteria } & \multicolumn{2}{|c|}{ Gram-negative bacteria } & \multirow{2}{*}{$\begin{array}{c}\text { Fungi } \\
\text { Candida } \\
\text { albicans ATTCC- } \\
10231\end{array}$} \\
\hline & $\begin{array}{c}\text { Staphylococcus aureus } \\
\text { ATTCC-29213 }\end{array}$ & $\begin{array}{c}\text { Bacilils } \\
\text { subtilis ATTCC- } \\
10400\end{array}$ & $\begin{array}{c}\text { Escherichia coli } \\
\text { ATTCC-35218 }\end{array}$ & $\begin{array}{c}\text { Pseudomonas } \\
\text { aeruginosa } \\
\text { ATTCC-29336 }\end{array}$ & \\
\hline $7 \mathbf{b}$ & N.A. & ++ & N.A. & N.A. & N.A. \\
\hline $7 d$ & N.A. & ++ & N.A. & N.A. & N.A. \\
\hline $7 e$ & N.A. & ++ & N.A. & N.A. & N.A. \\
\hline $7 f$ & N.A. & ++ & N.A. & N.A. & N.A. \\
\hline $7 \mathrm{~g}$ & N.A. & ++ & N.A. & N.A. & N.A. \\
\hline $8 e$ & N.A. & ++ & N.A. & N.A. & +++ \\
\hline $8 f$ & N.A. & ++++ & N.A. & N.A. & ++++ \\
\hline $8 g$ & N.A. & ++ & N.A. & N.A. & ++ \\
\hline Ciprofloxacin & +++ & ++++ & ++++ & ++++ & N.A. \\
\hline Ketoconazole & N.A. & N.A. & N.A. & N.A. & ++++ \\
\hline
\end{tabular}

$10^{8} \mathrm{CFU} / \mathrm{mL}$ of pathological tested bacteria, $1 \times 10^{6} \mathrm{CFU} / \mathrm{mL}$ of yeast and $1 \times 10^{4}$ spore $/ \mathrm{mL}$ of fungi spread on nutrient agar (NA), Sabouraud dextrose agar (SDA), and potato dextrose agar (PDA) medium, respectively. After the media had cooled and solidified, wells (10 $\mathrm{mm}$ in diameter) were made in the solidified agar and loaded with $100 \mathrm{~mL}$ of tested compound solution prepared by dissolving $100 \mathrm{mg}$ of the chemical compound in one $\mathrm{mL}$ of dimethyl sulfoxide (DMSO). The inculcated plates were then incubated for $24 \mathrm{~h}$ at $37^{\circ} \mathrm{C}$ for bacteria and $48 \mathrm{~h}$ at $28^{\circ} \mathrm{C}$ for fungi. Negative controls were prepared using DMSO employed for dissolving the tested compound. Ciprofloxacin $(50 \mathrm{mg} / \mathrm{mL})$ and Ketoconazole $(50 \mathrm{mg} / \mathrm{mL})$ were used as standard for antibacterial and antifungal activity, respectively. After incubation time, antimicrobial activity was evaluated by measuring the zone of inhibition against the test organisms and compared with that of the standard. The observed zone of inhibition is presented in Table 1. Antimicrobial activities were expressed as inhibition diameter zones in millimeters $(\mathrm{mm})$ as follows: N.A. (no activity $\leq 4 \mathrm{~mm} ;+($ weak $)=5-9 \mathrm{~mm} ;++($ moderate $)=10-$ $15 \mathrm{~mm} ;+++($ strong $)=16-20 \mathrm{~mm}$, and $++++($ very strong $) \geq$
$21 \mathrm{~mm}$. The experiment was carried out in triplicate and the average zone of inhibition was calculated.

4.1. Antimicrobial Activity. A sample of some synthesized compounds (7b, 7d, 7e, 7f and 8e, 8f, 8g) has been subjected to antimicrobial activity studies including Gram-positive bacteria (Bacillus subtilis and Staphylococcus aureus), Gramnegative bacteria (Pseudomonas Aeruginosa and Escherichia coli), and fungi (Candida albicans). Antimicrobial tests were carried out by the agar well diffusion method. When compared to the standard drug Ciprofloxacin, it was seen that compounds $\mathbf{7 b}, \mathbf{7 d}, \mathbf{7 e}$, and $\mathbf{7 f}$ with frame structure of indole moiety showed an inhibition effect against Bacillus subtilis ATCC 10400 (Table 4). On the other hand, 8e, 8g with frame structure of pyrrole moiety showed effect against Bacillus subtilis ATCC 10400. Not worthy to mention that $\mathbf{8 f}$ showed potent inhibition against the same Gram-positive bacteria Bacillus subtilis ATCC 10400 compared with standard Ciprofloxacin (Table 4). Interestingly, $\mathbf{8 e}, \mathbf{8 g}$ with frame structure of pyrrole moiety showed antifungal activity against Candida albicans ATTCC-10231, while 8f showed potent 
inhibition against the same fungi Candida albicans ATTCC1023 compared to standard drug Ketoconazole. Nevertheless, $\mathbf{7 b}, \mathbf{7 d}, \mathbf{7 e}, \mathbf{7 f}, \mathbf{8 e}, \mathbf{8 f}$, and $\mathbf{8 g}$ were not active against $S$. aureus ATCC 29213, E. coli ATCC-35218, and P. aeruginosa ATCC 29336. The results obtained are summarized in Table 4 [59].

\section{Conclusion}

In summary, Feist's acid has been introduced as a new class of hydrogen bond donor catalysts for the activation of nitroalkene in conjugate addition reactions. This study includes the original report of Feist's acid catalysis of Michel addition of indole and pyrrole to a variety of nitroolefins. All the synthesized indole and pyrrole derivatives have be screened for antimicrobial activity (MIC determination) in our laboratory and the results are reported here in. Investigations surrounding the potential associated with Feist's acid catalysis, including the development of enantioselective variants, as a new tool for organic synthesis are under progress in our laboratory.

\section{Conflict of Interests}

The authors declare that there is no conflict of interests regarding the publication of this paper.

\section{Acknowledgment}

The authors extend their appreciation to the Deanship of Scientific Research at King Saud University for funding the work through the research group Project no. RGP-VPP-044.

\section{References}

[1] B. M. Trost, "On inventing reactions for atom economy," Accounts of Chemical Research, vol. 35, no. 9, pp. 695-705, 2002.

[2] B. M. Trost, "The atom economy-a search for synthetic efficiency," Science, vol. 254, no. 5037, pp. 1471-1477, 1991.

[3] E. Marqués-López, A. Diez-Martinez, P. Merino, and R. P. Herrera, "The role of the indole in important organocatalytic enantioselective Friedel-Crafts alkylation reactions," Current Organic Chemistry, vol. 13, no. 16, pp. 1585-1609, 2009.

[4] S.-L. You, Q. Cai, and M. Zeng, "Chiral Brønsted acid catalyzed Friedel-Crafts alkylation reactions," Chemical Society Reviews, vol. 38, no. 8, pp. 2190-2210, 2009.

[5] V. Terrasson, R. M. De Figueiredo, and J. M. Campagne, "Organocatalyzed asymmetric Friedel-Crafts reactions," European Journal of Organic Chemistry, vol. 2010, no. 14, pp. 26352655, 2010.

[6] M. Zeng and S.-L. You, "Asymmetric Friedel-Crafts alkylation of indoles: the control of enantio- and regioselectivity," Synlett, vol. 2010, no. 9, pp. 1289-1301, 2010.

[7] N. Ono, The Nitro Group in Organic Synthesis, John Wiley \& Sons, New York, NY, USA, 2001.

[8] D. Seebach, E. W. Colvin, F. Lehr, and T. Weller, "Nitroaliphatic compounds-ideal intermediates in organic synthesis," Chimia, vol. 33, pp. 1-18, 1979.
[9] G. Calderari and D. Seebach, "Asymmetric Michael additions. Stereoselective alkylation of chiral, non-racemic enolates by nitroolefins. Preparation of enantiomerically pure $\gamma$ aminobutyric acid and succinic acid derivatives," Helvetica Chimica Acta, vol. 68, no. 6, pp. 1592-11604, 1985.

[10] O. M. Berner, L. Tedeschi, and D. Enders, "Asymmetric Michael additions to nitroalkenes," European Journal of Organic Chemistry, vol. 2002, no. 12, pp. 1877-1894, 2002.

[11] M. Chakrabarty, R. Basak, and N. Ghosh, "Microwave-assisted Michael reactions of 3-( $2^{\prime}$-nitrovinyl)indole with indoles on TLC-grade silica gel. A new, facile synthesis of 2,2-bis $\left(3^{\prime}\right.$ indolyl)nitroethanes," Tetrahedron Letters, vol. 42, no. 23, pp. 3913-3915, 2001.

[12] B. Wang, "Sulfamic acid: a very useful catalyst," Synlett, vol. 2005, no. 8, pp. 1342-1343, 2005.

[13] S. Sulzer-Mossé and A. Alexakis, "Chiral amines as organocatalysts for asymmetric conjugate addition to nitroolefins and vinyl sulfones via enamine activation," Chemical Communications, no. 30, pp. 3123-3135, 2007.

[14] D. Almaşi, D. A. Alonso, and C. N. Nájera, "Organocatalytic asymmetric conjugate additions," Tetrahedron: Asymmetry, vol. 18, no. 3, pp. 299-365, 2007.

[15] S. B. Tsogoeva, "Recent advances in asymmetric organocatalytic 1,4-conjugate additions," European Journal of Organic Chemistry, vol. 2007, no. 11, pp. 1701-1716, 2007.

[16] D. Roca-López, D. Sadaba, I. Delso, R. P. Herrera, T. Tejero, and P. Merino, "Asymmetric organocatalytic synthesis of $\gamma$ nitrocarbonyl compounds through Michael and Domino reactions," Tetrahedron: Asymmetry, vol. 21, no. 21-22, pp. 2561-2601, 2010.

[17] R. P. Herrera, V. Sgarzani, L. Bernardi, and A. Ricci, "Catalytic enantioselective Friedel-Crafts alkylation of indoles with nitroalkenes by using a simple thiourea organocatalyst," Angewandte Chemie, vol. 44, no. 40, pp. 6576-6579, 2005.

[18] E. M. Fleming, T. McCabe, and S. J. Connon, "Novel axially chiral bis-arylthiourea-based organocatalysts for asymmetric Friedel-Crafts type reactions," Tetrahedron Letters, vol. 47, no. 39, pp. 7037-7042, 2006.

[19] M. Ganesh and D. Seidel, "Catalytic enantioselective additions of indoles to nitroalkenes," Journal of the American Chemical Society, vol. 130, no. 49, pp. 16464-16465, 2008.

[20] J. Itoh, K. Fuchibe, and T. Akiyama, "Chiral phosphoric acid catalyzed enantioselective Friedel-Crafts alkylation of indoles with nitroalkenes: cooperative effect of $3 \AA$ molecular sieves," Angewandte Chemie, vol. 47, no. 21, pp. 4016-4018, 2008.

[21] E. Marqués-López, A. Alcaine, T. Tejero, and R. P. Herrera, "Enhanced efficiency of thiourea catalysts by external Brønsted acids in the Friedel-Crafts alkylation of indoles," European Journal of Organic Chemistry, vol. 2011, no. 20-21, pp. 37003705, 2011.

[22] G. A. Olah, R. Krishnamurty, and G. K. S. Prakash, "FriedelCrafts alkylation," in Comprehensive Organic Synthesis, B. M. Trost and I. Fleming, Eds., vol. 3 of Comprehensive Organic Synthesis, p. 293, Pergamon Press, Oxford, UK, 1st edition, 1991.

[23] I. Komoto and S. Kobayashi, "Lewis acid catalysis in supercritical carbon dioxide. Use of poly(ethylene glycol) derivatives and perfluoroalkylbenzenes as surfactant molecules which enable efficient catalysis in $\mathrm{ScCO}_{2}$," Journal of Organic Chemistry, vol. 69, no. 3, pp. 680-688, 2004.

[24] Z.-P. Zhan, R.-F. Yang, and K. Lang, "Samarium triiodidecatalyzed conjugate addition of indoles with electron-deficient 
olefins," Tetrahedron Letters, vol. 46, no. 22, pp. 3859-3862, 2005.

[25] Y.-X. Jia, S.-F. Zhu, Y. Yang, and Q.-L. Zhou, "Asymmetric Friedel-Crafts alkylations of indoles with nitroalkenes catalyzed by Zn(II)-bisoxazoline complexes," Journal of Organic Chemistry, vol. 71, no. 1, pp. 75-80, 2006.

[26] P. M. Pihko, Hydrogen Bonding in Organic Synthesis, John Wiley \& Sons, Weinheim, Germany, 2009.

[27] A. G. Doyle and E. N. Jacobsen, "Small-molecule H-bond donors in asymmetric catalysis," Chemical Reviews, vol. 107, no. 12, pp. 5713-5743, 2007.

[28] T. Akiyama, "Stronger Brønsted acids," Chemical Reviews, vol. 107, no. 12, pp. 5744-5758, 2007.

[29] Y. Takemoto, "Recognition and activation by ureas and thioureas: stereoselective reactions using ureas and thioureas as hydrogen-bonding donors," Organic and Biomolecular Chemistry, vol. 3, no. 24, pp. 4299-4306, 2005.

[30] Z. Zhang and P. R. Schreiner, "(Thio)urea organocatalysiswhat can be learnt from anion recognition?" Chemical Society Reviews, vol. 38, no. 4, pp. 1187-1198, 2009.

[31] S. J. Connon, "The design of novel, synthetically useful (thio)urea-based organocatalysts," Synlett, vol. 2009, no. 3, pp. 354-376, 2009.

[32] N. T. Tran, S. O. Wilson, and A. K. Franz, "Cooperative hydrogen-bonding effects in silanediol catalysis," Organic Letters, vol. 14, no. 1, pp. 186-189, 2012.

[33] A. G. Schafer, J. M. Wieting, and A. E. Mattson, "Silanediols: a new class of hydrogen bond donor catalysts," Organic Letters, vol. 13, no. 19, pp. 5228-5231, 2011.

[34] A. M. A. Shumaila and R. S. Kusurkar, "Silica gel, an effective catalyst for the reaction of electron-deficient nitro-olefins with nitrogen heterocycles," Synthetic Communications, vol. 40, no. 19, pp. 2935-2940, 2010.

[35] J. F. Schneider, F. C. Falk, R. Freohlich, and J. Paradies, "Planarchiral thioureas as hydrogen-bond catalysts," European Journal of Organic Chemistry, vol. 2010, no. 12, pp. 2265-2269, 2010.

[36] M. Ganesh and D. Seidel, "Catalytic enantioselective additions of indoles to nitroalkenes," Journal of the American Chemical Society, vol. 130, no. 49, pp. 16464-16465, 2008.

[37] N. Takenaka, R. S. Sarangthem, and S. K. Seerla, "2Aminopyridinium ions activate nitroalkenes through hydrogen bonding," Organic Letters, vol. 9, no. 15, pp. 2819-2822, 2007.

[38] Y. Gu, C. Ogawa, and S. Kobayashi, "Silica-supported sodium sulfonate with ionic liquid: a neutral catalyst system for Michael reactions of indoles in water," Organic Letters, vol. 9, no. 2, pp. 175-178, 2007.

[39] G. Dessole, R. P. Herrera, and A. Ricci, "H-bonding organocatalysed Friedel-Crafts alkylation of aromatic and heteroaromatic systems with nitroolefins," Synlett, no. 13, pp. 2374-2378, 2004.

[40] W. Zhuang, R. G. Hazell, and K. A. Jørgensen, "Enantioselective Friedel-Crafts type addition of indoles to nitro-olefins using a chiral hydrogen-bonding catalyst-synthesis of optically active tetrahydro- $\beta$-carbolines," Organic and Biomolecular Chemistry, vol. 3, no. 14, pp. 2566-2571, 2005.

[41] C. Lin, J. Hsu, M. N. V. Sastry et al., " $I_{2}$-catalyzed Michael addition of indole and pyrrole to nitroolefins," Tetrahedron, vol. 61, no. 49, pp. 11751-11757, 2005.

[42] P. R. Schreiner, "Metal-free organocatalysis through explicit hydrogen bonding interactions," Chemical Society Reviews, vol. 32, no. 5, pp. 289-296, 2003.
[43] S. J. Connon, "Organocatalysis mediated by (thio)urea derivatives," Chemistry European Journal, vol. 12, no. 21, pp. 5418-5427, 2006.

[44] M. S. Taylor and E. N. Jacobsen, "Asymmetric catalysis by chiral hydrogen-bond donors," Angewandte Chemie, vol. 45, no. 10, pp. 1520-1543, 2006.

[45] Z. Zhang and P. R. Schreiner, "(Thio)urea organocatalysiswhat can be learnt from anion recognition?” Chemical Society Reviews, vol. 38, no. 4, pp. 1187-1198, 2009.

[46] W. Zhou, L.-W. Xu, L. Yang, P.-Q. Zhao, and C.-G. Xia, "Novel Brønsted acid-catalyzed Michael-type Friedel-Crafts reactions of indoles and acetalization of aldehydes," Journal of Molecular Catalysis A, vol. 249, no. 1-2, pp. 129-134, 2006.

[47] W. Zhou, L.-W. Xu, L. Li, L. Yang, and C.-G. Xia, "Enantioselective Michael-type Friedel-Crafts reactions of indoles to enones catalyzed by a chiral camphor-based Brønsted acid," European Journal of Organic Chemistry, vol. 2006, no. 23, pp. 5225-5227, 2006.

[48] S.-I. Kondo, T. Harada, R. Tanaka, and M. Unno, "Anion recognition by a silanediol-based receptor," Organic Letters, vol. 8, no. 20, pp. 4621-4624, 2006.

[49] F. R. Goss, C. K. Ingold, and J. F. Thorpe, "XLI.--the chemistry of the glutaconic acids. Part XIV. Three-carbon tautomerism in the cyclopropane series," Journal of the Chemical Society: Transactions, vol. 123, pp. 327-361, 1923.

[50] A. M. Al-Majid, B. L. Booth, and J. T. Gomes, " $\mathrm{C}_{2}$-symmetric ligands for asymmetric catalysis based on Feist's acid," Journal of Chemical Research: Part S, no. 2, pp. 78-79, 1998.

[51] R. K. Y. Zee-Cheng and C. C. Cheng, "Structure-activity relationship study of anthraquinones: 1, 4-dihydroxy-5, 8bis[[2-(2-hydroxyethoxy)ethyl]amino]-9, 10-anthracenedione, an analog of an established antineoplastic agent," Journal of Pharmaceutical Science, vol. 71, no. 6, pp. 708-709, 1982.

[52] F. Epifano, S. Genovese, L. Menghini, and M. Curini, "Chemistry and pharmacology of oxyprenylated secondary plant metabolites," Phytochemistry, vol. 68, no. 7, pp. 939-953, 2007.

[53] S. Khatib, O. Nerya, R. Musa, M. Shmuel, S. Tamir, and J. Vaya, "Chalcones as potent tyrosinase inhibitors: the importance of a 2,4-substituted resorcinol moiety," Bioorganic \& Medicinal Chemistry, vol. 13, no. 2, pp. 433-441, 2005.

[54] A. M. Almerico, P. Diana, P. Barraja et al., "Glycosidopyrroles. Part 1. Acyclic derivatives: 1-(2-hydroxyethoxy) methylpyrroles as potential anti-viral agents," IL Farmaco, vol. 53, no. 1, pp. 3340, 1998.

[55] H. Carpio, E. Galeazzi, R. Greenhouse et al., "Synthesis of 1, 2-dihydro-3H-pyrrolo[1, 2-a]pyrrole-1-carboxylic acids and homologous pyridine and azepine analogues thereof," Canadian Journal of Chemistry, vol. 60, no. 18, pp. 2295-2312, 1982.

[56] G. Dannhardt, W. Kiefer, G. Krämer, S. Maehrlein, U. Nowe, and B. Fiebich, "The pyrrole moiety as a template for COX-1/COX2 inhibitors," European Journal of Medicinal Chemistry, vol. 35, no. 5, pp. 499-510, 2000.

[57] M. A. Evans, D. C. Smith, J. M. Holub et al., "Synthesis and cytotoxicity of substituted ethyl 2-phenacyl-3-phenylpyrrole-4carboxylates," Archiv der Pharmazie, vol. 336, no. 3, pp. 181-190, 2003.

[58] J. Wu, X. Li, F. Wu, and B. Wan, "A new type of bis(sulfonamide)diamine ligand for a $\mathrm{Cu}(\mathrm{OTf})_{2}$-catalyzed asymmetric FriedelCrafts alkylation reaction of indoles with nitroalkenes," Organic Letters, vol. 13, no. 18, pp. 4834-4837, 2011. 
[59] J. G. Collee, J. P. Duguid, A. G. Fraser, B .P. Marmion, and A. C. Scott, "Laboratory control of antimicrobial therapy," in Mackie and MacCartney Practical Medical Microbiology, vol. 2, pp. 161181, Churchill Livingstone, London, UK, 13th edition, 1989.

[60] N. A. Lange and W. E. Hambourger, "Condensation of aromatic aldehydes with nitromethane in the presence of alcoholic sodium hydroxide," Journal of the American Chemical Society, vol. 53, no. 10, pp. 3865-3867, 1931. 

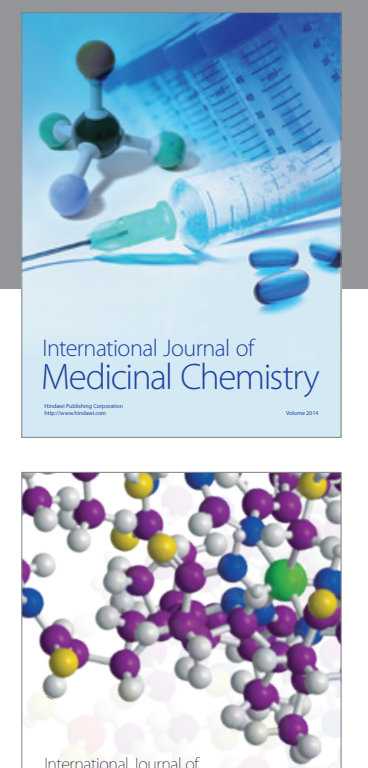

\section{Carbohydrate} Chemistry

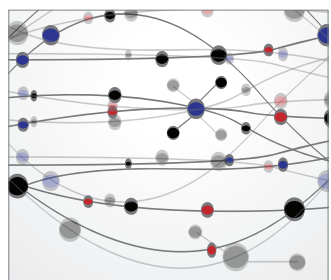

The Scientific World Journal
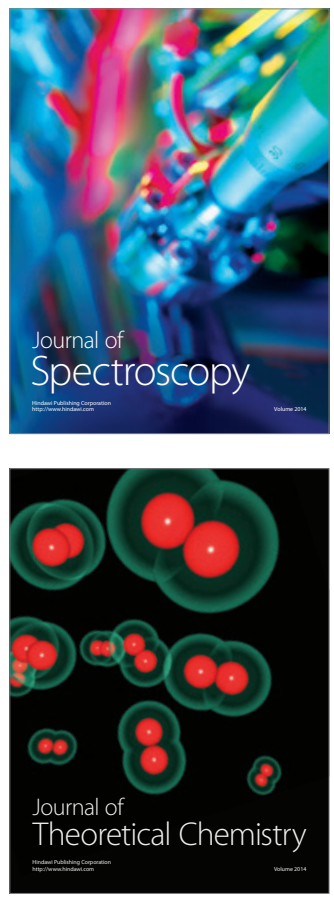
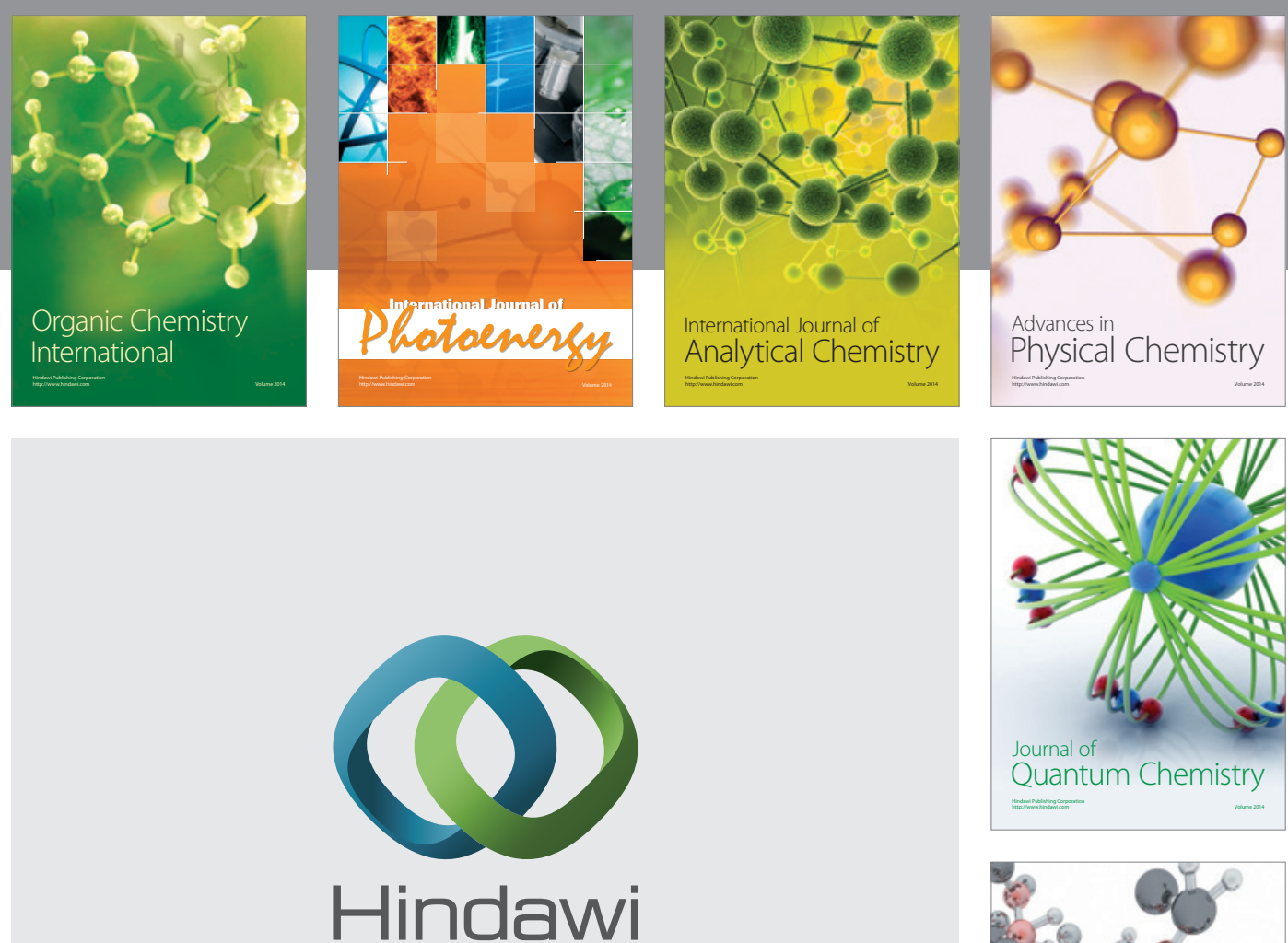

Submit your manuscripts at

http://www.hindawi.com

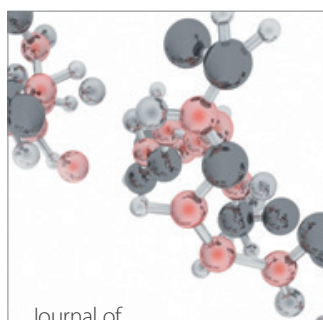

Analytical Methods

in Chemistry

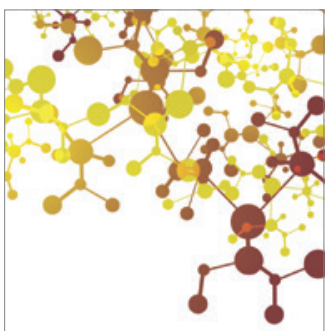

Journal of

Applied Chemistry

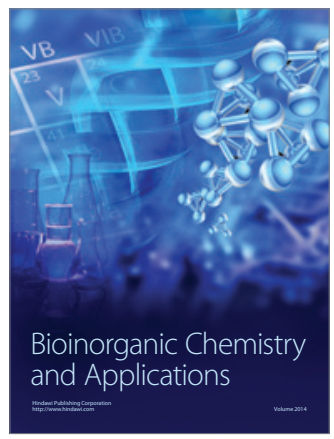

Inorganic Chemistry
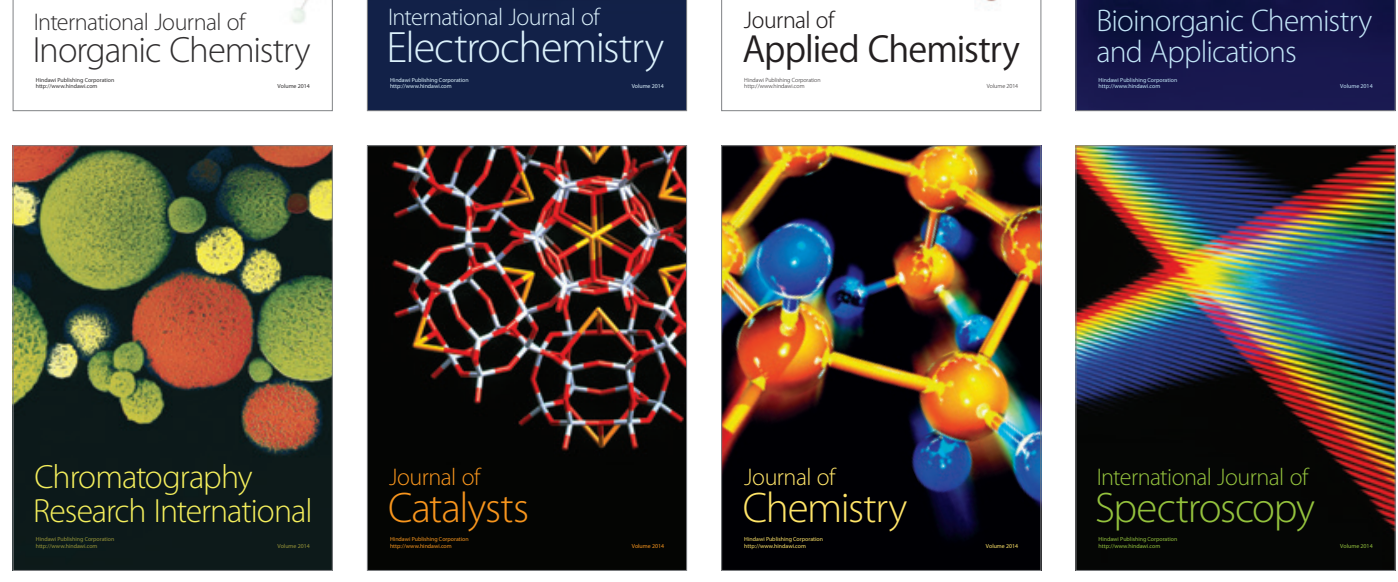